\title{
Identification of the Myogenetic Oligodeoxynucleotides (myoDNs) That Promote Differentiation of Skeletal Muscle Myoblasts by Targeting Nucleolin
}

\author{
Sayaka Shinji ${ }^{1}$, Koji Umezawa ${ }^{2,3}$, Yuma Nihashi ${ }^{4}$, Shunichi Nakamura ${ }^{1}$, \\ Takeshi Shimosato ${ }^{1,2,3,4}$ and Tomohide Takaya ${ }^{1,2,3,4 *}$ \\ ${ }^{1}$ Department of Agriculture, Graduate School of Science and Technology, Shinshu University, Nagano, Japan, ${ }^{2}$ Department \\ of Agricultural and Life Science, Faculty of Agriculture, Shinshu University, Nagano, Japan, ${ }^{3}$ Department of Biomolecular \\ Innovation, Institute for Biomedical Sciences, Shinshu University, Nagano, Japan, ${ }^{4}$ Department of Science and Technology, \\ Graduate School of Medicine, Science and Technology, Shinshu University, Nagano, Japan
}

OPEN ACCESS

Edited by:

Yuanyuan Yu,

Hong Kong Baptist University,

Hong Kong

Reviewed by:

Fangfei $\mathrm{Li}$

Hong Kong Baptist University,

Hong Kong

Halesha Dhurvigere Basavarajappa,

City of Hope, United States

${ }^{*}$ Correspondence:

Tomohide Takaya

ttakaya@shinshu-u.ac.jp

Specialty section:

This article was submitted to

Molecular Medicine,

a section of the journal

Frontiers in Cell and Developmental

Biology

Received: 13 October 2020 Accepted: 07 December 2020

Published: 11 January 2021

Citation:

Shinji S, Umezawa K, Nihashi Y,

Nakamura S, Shimosato $T$ and Takaya T (2021) Identification of the Myogenetic Oligodeoxynucleotides (myoDNs) That Promote Differentiation

of Skeletal Muscle Myoblasts by Targeting Nucleolin.

Front. Cell Dev. Biol. 8:616706 doi: 10.3389/fcell.2020.616706
Herein we report that the 18-base telomeric oligodeoxynucleotides (ODNs) designed from the Lactobacillus rhamnosus GG genome promote differentiation of skeletal muscle myoblasts which are myogenic precursor cells. We termed these myogenetic ODNs (myoDNs). The activity of one of the myoDNs, iSN04, was independent of Toll-like receptors, but dependent on its conformational state. Molecular simulation and iSN04 mutants revealed stacking of the 13-15th guanines as a core structure for iSN04. The alkaloid berberine bound to the guanine stack and enhanced iSNO4 activity, probably by stabilizing and optimizing iSNO4 conformation. We further identified nucleolin as an iSN04-binding protein. Results showed that iSNO4 antagonizes nucleolin, increases the levels of p53 protein translationally suppressed by nucleolin, and eventually induces myotube formation by modulating the expression of genes involved in myogenic differentiation and cell cycle arrest. This study shows that bacterial-derived myoDNs serve as aptamers and are potential nucleic acid drugs directly targeting myoblasts.

Keywords: oligodeoxynucleotide (ODN), berberine (CID: 12457), nucleolin (NCL), skeletal muscle myoblast, myogenic differentiation

\section{INTRODUCTION}

Skeletal muscle myoblasts are myogenic precursor cells that play a central role during muscle development and regeneration. In the first step of these processes, muscle stem cells called satellite cells on myofibers are activated into myoblasts. After several rounds of division, myoblasts differentiate into myocytes, which is led by myogenic transcription factors such as MyoD and myogenin. Myocytes then fuse to form multi-nuclear myotubes to generate or restore myofibers (Dumont et al., 2015). However, the differentiation ability of myoblasts declines due to aging or diseases. Aged murine myoblasts tend to differentiate into the fibrogenic lineage by the activation of the canonical Wnt pathway (Brack et al., 2007). The myoblasts isolated from the chronic kidney disease mice model showing muscle atrophy display attenuated MyoD expression and myotube formation (Zhang et al., 2010). Cancer-conditioned media inhibit myogenic differentiation 
by upregulating $\mathrm{C} / \mathrm{EBP} \beta$ in the murine myoblast cell line $\mathrm{C} 2 \mathrm{C} 12$ (Marchildon et al., 2015). Such hypoactivities of myoblasts are considered to be one of the reasons for the development of muscle atrophy (Fukada, 2018; McCormick and Vasilaki, 2018). Therefore, myoblast differentiation can be a clinical target for sarcopenia, disease-related muscle wasting, and cancer cachexia which are risk factors for mortality (Anker et al., 1997; Rubin, 2003; Carrero et al., 2008).

Several molecules have been identified that facilitate myogenic differentiation. Histone deacetylase inhibitors (HDACIs), trichostatin A (TSA), and valproic acid (VPA), promote myotube formation by inducing follistatin in myoblasts (Iezzi et al., 2004). However, TSA and VPA are non-specific HDACIs that affect a broad range of biological processes in vivo. Recent studies reported that the combined treatment of ursolic acid (UA) with leucine (Kim et al., 2015), and a single treatment of an oleanolic acid (OA) derivative (Cui et al., 2019) potentiates differentiation of $\mathrm{C} 2 \mathrm{C} 12$ cells. As the half-lives of UA in plasma and OA in serum are $<1 \mathrm{~h}$ (Chen et al., 2011; Li et al., 2012), their pharmacokinetic parameters need to be improved for clinical application.

Nucleic acids have tremendous potential for use in nextgeneration drugs. They are chemically synthesized, stable, and modifiable molecules that can access diverse targets with high specificities. Complementary antisense oligonucleotides modulate gene expression by degrading mRNAs, trapping microRNAs, or correcting splicing events (Quemener et al., 2020). Other types of oligonucleotides serve as aptamers that specifically interact with their target proteins (Wang et al., 2019). Furthermore, many immunomodulatory oligodeoxynucleotides (ODNs) from microbial and autologous DNA sequences have been reported. ODNs with unmethylated CpG motifs (CpGODNs) serve as ligands for Toll-like receptor (TLR) 9 and initiate inflammatory cascades (Vollmer and Krieg, 2009). In contrast, inhibitory ODNs (iODNs) representatively expressing telomeric elements suppress immunological reactions depending on TLR3, TLR7, and TLR9 (Klinman et al., 2008; Sackesen et al., 2013). At present, CpG-ODNs and iODNs are anticipated to be effective drugs for sepsis and allergic diseases (Wang Y. et al., 2015; Yamamoto et al., 2017).

Intriguingly, some CpG-ODNs have been reported to alter cell fate. Initial studies have shown that CpG-1826 modulates osteoclastogenesis through TLR9 (Zou et al., 2003; Amcheslavsky et al., 2005). CpG-KSK13 displayed an inhibitory effect on osteoclast differentiation by downregulating TREM-2 (Chang et al., 2009). CpG-2006 and its variants interfere with osteoblast differentiation from mesenchymal stem cells (MSCs) by inhibiting the BMP-Smad signal in a TLR9-independent manner (Norgaard et al., 2010). By contrast, MT01, a 27-base C-rich iODN (Yang et al., 2010), stimulates the differentiation of MSCs into osteoblasts via the ERK-p38 pathway (Feng et al., 2011; Hou et al., 2012; Shen et al., 2012).

These findings prompted us to explore a novel ODN that regulates myoblast differentiation. We recently constructed 18-base ODN candidates designed from the genome sequence of a lactic acid bacteria strain, Lactobacillus rhamnosus GG (LGG) (Nigar et al., 2017). In this study, these synthetic phosphorothioated (PS)-ODNs resistant to nucleases were applied to myoblasts to validate their myogenetic effects. Herein, we report a series of 18-base telomeric PSODNs, named myogenetic ODNs (myoDNs), that promote myoblast differentiation depending on their conformation but independent of TLR signal. This study presents an innovative approach to regulate cell fate using bacterial-derived ODNs.

\section{MATERIALS AND METHODS ODNs and Chemicals}

The sequences of the ODNs used in this study are described in Supplementary Table 1. PS-ODNs, 6-carboxyfluorescein (6-FAM)-conjugated PS-ODNs, and biotin-conjugated PSODNs were synthesized and purified via HPLC (GeneDesign, Osaka, Japan). AS1411 having a phosphodiester backbone was synthesized and desalted (Integrated DNA Technologies, Coralville, IA, USA) as previously reported (Girvan et al., 2006). PS-ODNs, AS1411, berberine hydrochloride (Nacalai, Osaka, Japan), palmatine chloride hydrate (Nacalai), coptisine chloride (Wako, Osaka, Japan), and jatrorrhizine (Wako) were dissolved in endotoxin-free water. An equal volume of endotoxin-free water instead of PS-ODNs and berberine analogs served as negative controls.

\section{Cell Culture}

All cells were cultured at $37^{\circ} \mathrm{C}$ under $5 \% \mathrm{CO}_{2}$ throughout the experiments.

Murine myoblasts (mMBs) were isolated from the skeletal muscle of 4-week-old C57BL/6J mice (Clea Japan, Tokyo, Japan) and primary-cultured as previously described (Takaya et al., 2017; Nihashi et al., 2019b). mMBs were maintained on the dishes or plates coated with collagen type I-C (Cellmatrix; Nitta Gelatin, Osaka, Japan), and cultured in growth medium (GM) for $\mathrm{mMB}$ consisting of Ham's F10 medium (Thermo Fisher Scientific, MA, USA), 20\% fetal bovine serum (FBS) (HyClone; GE Healthcare, UT, USA), $2 \mathrm{ng} / \mathrm{ml}$ recombinant human basic fibroblast growth factor (Wako), and a mixture of 100 units/ml penicillin and $100 \mu \mathrm{g} / \mathrm{ml}$ streptomycin (PS) (Nacalai).

Primary-cultured human myoblast (hMB) stock of adult healthy female (CC-2580; Lonza, MD, USA) was maintained according to the manufacturer's instruction. hMBs were seeded on collagen-coated dishes, cultured in Skeletal Muscle Growth Media-2 (CC-3245; Lonza) as GM for hMB, and differentiation was induced in differentiation medium (DM) for hMB consisting of DMEM (Nacalai) with $2 \%$ horse serum (HS) (HyClone; GE Healthcare) and PS.

C2C12 cells (DS Pharma Biomedical, Osaka, Japan) were seeded on collagen-coated dishes, cultured in GM for $\mathrm{C} 2 \mathrm{C} 12$ cells consisting of DMEM with 10\% FBS and PS, and induced differentiation in DM for C2C12 cells consisting of DMEM with $2 \% \mathrm{HS}$ and PS.

Murine embryonic fibroblasts (MEFs) were prepared from E12 embryos of Slc:ICR mice (Japan SLC, Shizuoka, Japan). The embryos that were removed their heads and internal organs were minced in GM for MEFs consisting of DMEM with 10\% FBS and PS. The tissue clusters were seeded and cultured for 3 days. Then 
outgrown cells were dissociated to single cells as MEFs using $0.25 \%$ trypsin with $1 \mathrm{mM}$ EDTA (Wako).

MC3T3-E1 cells (RCB1126) were provided by RIKEN BRC (Tsukuba, Japan) through the Project for Realization of Regenerative Medicine and the National Bio-Resource Project of the MEXT, Japan. The cells were maintained in EMEM (Wako) with $10 \%$ FBS and PS.

\section{Immunocytochemistry}

Immunocytochemistry of myoblasts was performed as previously described (Takaya et al., 2017; Nihashi et al., 2019a,b). The myoblasts were fixed with $2 \%$ paraformaldehyde, permeabilized with $0.2 \%$ Triton X-100 (Nacalai), and immunostained with $0.5 \mu \mathrm{g} / \mathrm{ml}$ mouse monoclonal anti-myosin heavy chain (MHC) antibody (MF20; R\&D Systems, MN, USA) and $1.0 \mu \mathrm{g} / \mathrm{ml}$ rabbit polyclonal anti-nucleolin antibody (ab22758; Abcam, Cambridge, UK). $0.1 \mu \mathrm{g} / \mathrm{ml}$ each of Alexa Fluor 488-conjugated donkey polyclonal anti-mouse IgG antibody and Alexa Fluor 594-conjugated donkey polyclonal anti-rabbit IgG antibody (Jackson ImmunoResearch, PA, USA) were used as secondary antibodies. Cell nuclei were stained with DAPI (Nacalai). Highresolution fluorescent images were taken under an EVOS FL Auto microscope (AMAFD1000; Thermo Fisher Scientific). The ratio of $\mathrm{MHC}^{+}$cells was defined as the number of nuclei in the $\mathrm{MHC}^{+}$cells divided by the total number of nuclei, and the fusion index was defined as the number of nuclei in the multinuclear $\mathrm{MHC}^{+}$myotubes divided by the total number of nuclei using ImageJ software (National Institutes of Health, USA).

\section{Screening System}

$1.0 \times 10^{4} \mathrm{mMBs}$ or $5.0 \times 10^{3} \mathrm{hMBs}$ in $100 \mu \mathrm{l} \mathrm{GM} /$ well were seeded on collagen-coated 96 -well plates. The next day, the medium was replaced with GM for $\mathrm{mMB}$ or $\mathrm{DM}$ for $\mathrm{hMB}$ containing PS-ODNs. After $48 \mathrm{~h}$, the myoblasts were subjected to MHC and DAPI staining. Fluorescent images were automatically captured using CellInsight NXT (Thermo Fisher Scientific). The ratio of $\mathrm{MHC}^{+}$cells of $\mathrm{mMBs}$ and $\mathrm{MHC}$ signal intensities of hMBs were automatically measured using HCS Studio: Cellomics Scan software (Thermo Fisher Scientific). The average value of three wells (four fields/well) served as the mean of each sample.

\section{Cell Counting}

$5.0 \times 10^{4} \mathrm{mMBs} /$ well were seeded on collagen-coated 24-well plates and $5.0 \times 10^{4} \mathrm{MEF} /$ well were seeded on 12 -well plates. The next day, the medium was replaced with medium containing 1 or $3 \mu \mathrm{M}$ iSN04. The cells were continuously cultured until cell counting. For counting, the cells were completely dissociated using $0.25 \%$ trypsin with $1 \mathrm{mM}$ EDTA and the number of cells was counted using a hemocytometer.

\section{Quantitative Real-Time RT-PCR (qPCR)}

$2.5 \times 10^{5} \mathrm{hMBs}$ in GM were seeded on collagen-coated 60$\mathrm{mm}$ dishes. The next day, the medium was replaced with DM containing $30 \mu \mathrm{M}$ iSN04. After $24 \mathrm{~h}$, total RNA of the hMBs was isolated using NucleoSpin RNA Plus (MachereyNagel, Düren, Germany) and was reverse transcribed using ReverTra Ace qPCR RT Master Mix (TOYOBO, Osaka, Japan). qPCR was performed using GoTaq qPCR Master Mix
(Promega, WI, USA) with StepOne Real-Time PCR System (Thermo Fisher Scientific). The amount of each transcript was normalized to that of tyrosine 3-monooxygenase/tryptophan 5monooxygenase activation protein zeta (YWHAZ) gene. The results are presented as fold-change. Primer sequences are listed in Supplementary Table 2.

\section{RNA Sequencing (RNA-Seq)}

The total RNA of hMBs used for qPCR was subjected to RNAseq (Novogene, Beijing, China). RNA quality was checked using an Agilent 2100 Bioanalyzer (Agilent Technologies, Waldbronn, Germany). RNA integrity number (RIN) values were 10.0 ( $\max$ score) in all samples (Supplementary Figure 4A). The RNA was subjected to library preparation using Illumina TruSeq RNA and DNA Sample Prep Kits (Illumina, CA, USA). Library quality was confirmed using a Qubit 2.0 fluorometer (Life Technologies; Thermo Fisher Scientific) and Agilent 2100 Bioanalyzer. RNAseq was performed using Illumina NovaSeq 6000 (Illumina) to generate $>6-\mathrm{GB}$ raw data per sample. Raw data were recorded in FASTQ format. The quality of the read was calculated as the arithmetic mean of the Phred quality score. The reads with following characteristics were discarded: adapter contamination, when uncertain nucleotides constituted $>10 \%$ of either read, or when low quality nucleotides (base quality $<20$ ) constituted $>50 \%$ of the read. The cleaned reads were mapped to a human reference genome (GRCh38.82) using TopHat2 (Kim et al., 2013). The number of the reads and mapping efficiencies are summarized in Supplementary Table 3. Expression levels of the transcripts were calculated as fragments per kilobase per million reads (FPKM) values using HTSeq (Anders et al., 2015). False discovery rate (FDR) was employed to correct their $p$-values. FASTQ raw read data of RNA-seq were deposited in the DDBJ Sequence Read Archive (DRA; Research Organization of Information and Systems, National Institute of Genetics, Mishima, Japan) with the accession number: DRA008498.

\section{Heatmap}

Heatmaps of FPKM values were generated via Heatmapper (http://www.heatmapper.ca/) (Babicki et al., 2016) with the following settings: Clustering method, Average linkage; Distance measurement method, Pearson.

\section{Gene Ontology (GO) Analysis}

The iSN04-dependent differentially expressed genes (DEGs) were subjected to GO analysis using DAVID Bioinformatics Resources 6.8 (https://david.ncifcrf.gov/) (Huang et al., 2009). The GO terms in biological processes and the KEGG pathways with $p<0.005$ and $<0.05$, respectively, were defined as significantly enriched gene clusters. Scatter plots of the DEGs were visualized using $\mathrm{R}$ software (R Development Core Team) with a Bioconductor package, Reactome Pathway Analysis (Yu and $\mathrm{He}, 2016)$.

\section{STRING Analysis}

Functional and physiological interactions of the iSN04dependent DEGs were visualized using STRING version 11.0 (https://string-db.org/) (Szklarczyk et al., 2019). 


\section{Basic Local Alignment Search Tool (BLAST) Search}

Homologous sequences of iSN04 in the genomes of humans (taxid: 9605) and mice (taxid: 10088) were searched and scored using BLAST (https://blast.ncbi.nlm.nih.gov/Blast.cgi).

\section{Trivial Trajectory Parallelization of Multicanonical Molecular Dynamics (TTP-McMD)}

Starting with the simulation of a single-chain iSN04 structure built from its DNA sequence by NAB in AmberTools (Macke and Case, 1998), enhanced ensemble method, TTP-McMD (Ikebe et al., 2011) was conducted, to sample the equilibrated conformations at $310 \mathrm{~K}$. In the TTP-McMD, the energy range of the multicanonical ensemble covered a temperature range from 280 to $380 \mathrm{~K}$. Sixty trajectories were used and the production run was conducted for $40 \mathrm{~ns}$ in each trajectory (total $2.4 \mu \mathrm{s}$ ). Throughout the simulation, the force field of amber ff12SB (Maier et al., 2015) was used for iSN04, whereas the solvation effect was considered as a generalized-born model (Tsui and Case, 2000). The force field for the berberine molecule was constructed from the RESP charge assigned by the quantum mechanics result of the DFT method with B3-LYP/6-31G*, and the other parameters were taken from GAFF (Wang et al., 2004). In the initial structure of the iSN04-berberine system, a berberine molecule were put at a distance of $4 \mathrm{~nm}$ from iSN04. The conformation of the iSN04-berberine complex was calculated via TTP-McMD under the same conditions as the iSN04 simulation.

\section{Agarose Gel Electrophoresis}

$0.8 \mathrm{nmol}$ PS-ODNs and $0.8 \mathrm{nmol}$ berberine analogs were mixed in $16 \mu \mathrm{l}$ Ham's F10 medium (Supplementary Table 4). In the experiments shown in Supplementary Figure 5B, iSN04 and berberine were mixed in sterile water, or $4.5 \mathrm{mM}$ of $\mathrm{HCl}, \mathrm{NaCl}$, $\mathrm{MgCl}_{2}, \mathrm{KCl}, \mathrm{CaCl}_{2}$, or $\mathrm{MnCl}_{2}$ solution, or $0.45 \mathrm{mM}$ of $\mathrm{FeSO}_{4}$, $\mathrm{CuSO}_{4}$, or $\mathrm{ZnSO}_{4}$ solution. The mixtures were placed at $4^{\circ} \mathrm{C}$ overnight, and then subjected to agarose gel electrophoresis using a TAE-buffered $3 \%$ agarose gel with $0.5 \mu \mathrm{g} / \mathrm{ml}$ ethidium bromide (EtBr). For colored images, the gels were illuminated by $302 \mathrm{~nm}$ ultraviolet (UV) using a UV Transilluminator (UVP, CA, USA) and the images were captured by a digital still camera without any filters. For monochromatic images, the gels were illuminated by 365-nm UV and the images were taken using ImageQuant LAS 500 with an emission bandpass filter of $560 \mathrm{~nm}$ (GE Healthcare).

\section{Protein Precipitation, SDS-PAGE, and CBB Staining}

Soluble whole-cell lysates of $\mathrm{C} 2 \mathrm{C} 12$ and MC3T3-E1 cells were prepared using lysis buffer consisting of $0.1 \mathrm{M}$ Tris- $\mathrm{HCl}$ ( $\mathrm{pH} 7.4), 75 \mathrm{mM} \mathrm{NaCl}$, and $1 \%$ Triton $\mathrm{X}-100$ with protease inhibitor cocktail (1 mM 4-(2-aminoethyl)benzenesulfonyl fluoride hydrochloride, $0.8 \mu \mathrm{M}$ aprotinin, $15 \mu \mathrm{M}$ E-64, $20 \mu \mathrm{M}$ leupeptin hemisulfate monohydrate, $50 \mu \mathrm{M}$ bestatin, and $10 \mu \mathrm{M}$ pepstatin A) (Nacalai). The biotin-conjugated PS-ODNs were immobilized on streptavidin-coated magnetic beads (Magnosphere MS300/Streptavidin; JSR Life Sciences, CA,
USA) according to the manufacturer's instruction. One hundred microgram of lysates and $0.6 \mathrm{mg}$ of iSN14-beads were mixed in $1 \mathrm{ml}$ lysis buffer with $1 \% \mathrm{NP}-40$ (Nacalai), and then gently rotated at $4{ }^{\circ} \mathrm{C}$ overnight to eliminate the non-specific proteins absorbing onto ODNs or beads. After magnetic pull-down of iSN14-beads, the supernatants were admixed with iSN04-beads and rotated at $4{ }^{\circ} \mathrm{C}$ overnight. The proteins precipitated by iSN04-beads were dissociated in lysis buffer with $1 \%$ NP-40, 10\% glycerol, $2 \%$ sodium dodecyl sulfate (SDS) at $95^{\circ} \mathrm{C}$ for $5 \mathrm{~min}$. The supernatants were subjected to SDS-PAGE using an $8 \%$ polyacrylamide gel. The gel was subjected to CBB staining using CBB Stain One Super (Nacalai) and scanned using ImageQuant LAS 500 .

\section{Mass Spectrometry}

The proteins within the CBB-stained gel were identified by mass spectrometry (MS Bioworks, MI, USA). In-gel digestion was performed using the ProGest robot (Digilab, MA, USA). The gels were washed with $25 \mathrm{mM}$ ammonium bicarbonate followed by acetonitrile, reduced with $10 \mathrm{mM}$ dithiothreitol at $60^{\circ} \mathrm{C}$ followed by alkylation with $50 \mathrm{mM}$ iodoacetamide at room temperature, digested with trypsin (Promega) at $37^{\circ} \mathrm{C}$ for $4 \mathrm{~h}$, and quenched with formic acid. Then the supernatant was subjected to analysis by nano LC-MS/MS with a Waters NanoAcquity HPLC system interfaced to a Thermo Fisher Q Exactive. Peptides were loaded on a trapping column and eluted over a $75-\mu \mathrm{m}$ analytical column at $350 \mathrm{nl} / \mathrm{min}$. Both columns were packed with Luna C18 resin (Phenomenex, CA, USA). The mass spectrometer was operated in data-dependent mode, with the Orbitrap operating at 70,000 FWHM and 17,500 FWHM for MS and MS/MS, respectively. The 15 most abundant ions were selected for MS/MS analysis. Data were searched using a local copy of Mascot with the following parameters: Enzyme, trypsin/P; Database, SwissProt Mouse; Fixed modification, carbamidomethyl; Variable modifications, oxidation, acetyl, pyro-Glu, deamidation; Mass values, monoisotopic; Peptide mass tolerance, $10 \mathrm{ppm}$; Fragment mass tolerance, $0.02 \mathrm{Da}$; Max missed cleavages, 2. Mascot DAT files were parsed into Scaffold (Proteome Software, OR, USA) for validation, filtering, and to create a non-redundant list per sample. Data were filtered using $1 \%$ protein and peptide FDR, which required at least two unique peptides per protein.

\section{Western Blotting}

Soluble whole-cell lysates of the hMBs treated with $30 \mu \mathrm{M}$ of iSN04 or AS1411 in DM for $48 \mathrm{~h}$ were prepared as described above. The lysates were denatured with $50 \mathrm{mM}$ Tris- $\mathrm{HCl}(\mathrm{pH}$ 6.8), $10 \%$ glycerol, and $2 \% \mathrm{SDS}$ at $95^{\circ} \mathrm{C}$ for $5 \mathrm{~min}$. Ten microgram of protein samples were subjected to SDS-PAGE on a $10 \%$ polyacrylamide gel followed by Western blotting using an iBlot 2 Dry Blotting System (Thermo Fisher Scientific). $1.0 \mu \mathrm{g} / \mathrm{ml}$ each of rabbit polyclonal anti-nucleolin antibody, mouse monoclonal anti-p53 antibody (PAb 240; Abcam), and mouse monoclonal anti-glyceraldehyde 3-phosphate dehydrogenase (GAPDH) antibody (5A12; Wako) were used as primary antibodies. $0.1 \mu \mathrm{g} / \mathrm{ml}$ each of horseradish peroxidase (HRP)conjugated goat anti-rabbit and anti-mouse $\operatorname{IgG}$ antibodies 
(Jackson ImmunoResearch) were used as secondary antibodies, respectively. HRP activity was detected using ECL Prime reagents (GE Healthcare) and ImageQuant LAS 500. The quantities of nucleolin and p53 proteins were normalized to that of GAPDH using Image J software.

\section{Statistical Analyses}

Results are presented as the mean \pm standard error. Statistical comparisons were performed using unpaired two-tailed Student's t-test, multiple comparison test with Dunnett's test, TukeyKramer test, Scheffe's F-test, or Williams' test where appropriate following one-way analysis of variance using $\mathrm{R}$ software. Statistical significance was set to $p<0.05$.

\section{RESULTS}

\section{Identification of myoDNs}

Fifty 18-base PS-ODNs (iSN01-iSN50) (Supplementary Table 1) derived from the LGG genome were subjected to a screening system to investigate the effects on myogenic differentiation of primary-cultured mMBs. Ten micromolar PS-ODNs were administered to the mMBs maintained in GM for $48 \mathrm{~h}$. The mMBs were immunostained for MHC, a terminal differentiation marker of skeletal muscle (Supplementary Figure 1). The percentages of $\mathrm{MHC}^{+}$cells were automatically quantified in a non-biased manner. As shown in Figure 1A, seven PS-ODNs (iSN01-iSN07) significantly increased the ratio of $\mathrm{MHC}^{+}$ cells, but other PS-ODNs did not alter the differentiation of mMBs. iSN01-iSN07 reproducibly induced myogenic differentiation of another independently isolated lot of $\mathrm{mMBs}$ (Supplementary Figure 2A), regardless of variation in the basal differentiation efficiency. In both screening results, iSN04' exhibited the highest myogenetic activity. These experiments were performed using iSN04' (AAG TTA GGG TGA GGG TGA; not existing in the LGG genome) instead of iSN04 (A $\underline{\text { GA }}$ TTA GGG TGA GGG TGA; existing in the LGG genome). As the activities of iSN04' and iSN04 were completely equal (Supplementary Figure 2C), iSN04 was utilized in the following experiments. iSN04 also promoted myogenic differentiation of the murine myoblast cell line $\mathrm{C} 2 \mathrm{C} 12$ (Supplementary Figure 2D) and primary-cultured hMBs (Figure 1B). The ratio of $\mathrm{MHC}^{+}$myocytes and fused myotubes was significantly increased by iSN04 in myoblasts in both mice and humans.

In stem cells or their progenies, proliferation, and differentiation are inverse processes, which negatively regulate each other (Ruijtenberg and van den Heuvel, 2016). The numbers of mMBs treated with iSN01-iSN07 were significantly lower than that of the control (Supplementary Figure 2B), indicating that iSN01-iSN07 inhibit myoblast proliferation. Continuous cell counting revealed that iSN04 suppressed the growth of mMBs in a dose-dependent manner; however, iSN04 did not alter the number of MEFs (Figure 1C). This demonstrates that the reduction in cell numbers in the iSN04-treated myoblasts was due to enhanced myogenic differentiation. qPCR revealed that iSN04 significantly upregulated the levels of myogenic transcription factors MyoD (MYOD1) and myogenin (MYOG), resulting in marked induction of embryonic MHC (MYH3) in hMBs (Figure 1D). In contrast, iSN04 did not alter the levels of undifferentiated myoblast markers, Pax7 (PAX7) and Myf5 (MYF5). These data show that iSN04 inherently promotes myoblast differentiation by activating the myogenic gene expression program.

We designated iSN01-iSN07 as "myoDNs," denoting myogenetic ODNs. They are a novel type of ODNs that induce myoblast differentiation.

\section{myoDN Activity Is Independent of TLR Signaling}

iSN01-iSN07 share a tandem repeat of a telomeric hexamer (TTAGGG TGAGGG) (Supplementary Figure 2E). A previous study has reported that a 24-base telomeric iODN (Tel-ODN) (Supplementary Table 1) suppresses human B-cell activation depending on TLR3, TLR7, and TLR9 (Sackesen et al., 2013). We have indicated that a 17-base telomeric iODN (iSG3; CCTCA TTAGGG TGAGGG) inhibits CpG-ODN (MsST)induced interleukin (IL)-6 expression through intracellular incorporation in murine macrophages (Wang Y. et al., 2015). Administration of 6-FAM-conjugated iSN04 to hMBs also showed that iSN04 was internalized into the cytoplasm within $2 \mathrm{~h}$ (Figure 1E). Contrarily, we have already confirmed that iSN01iSN07 does not affect MsST-induced IL-6 expression in murine splenocytes (Nigar et al., 2017), suggesting that myoDNs are not iODNs. RT-PCR revealed that hMBs, mMBs, and $\mathrm{C} 2 \mathrm{C} 12$ cells expressed all TLR genes except for Tlr12 in C2C12 cells (Supplementary Figures 3A,B). To investigate the dependency of myoDN activity on TLR signaling, hMBs were treated with iSN04, Tel-ODN, or CpG-2006 (Supplementary Table 1). CpG2006 is the TLR9 ligand initiating inflammatory responses in human lymphocytes and murine macrophages (Pohar et al., 2015). In the absence of iSN04, neither CpG-2006 nor Tel$\mathrm{ODN}$ induced the differentiation of hMBs into $\mathrm{MHC}^{+}$myocytes (Figure 1F). In the presence of iSN04, neither CpG-2006 nor TelODN inhibited iSN04-induced myogenic differentiation. RNAseq data (see next section) showed that transcription levels of the genes involved in the TLR signaling pathway were not altered in the iSN04-treated hMBs (Supplementary Figure 3C). These results demonstrate that iSN04 is not a TLR ligand, dissimilar to immunogenic CpG-ODNs and iODNs. It is assumed that myoDN activity inducing myogenic differentiation is independent of TLR signaling.

\section{Profile of iSN04-Dependent Gene Expression}

We comprehensively surveyed the iSN04-dependent gene expression profile of hMBs. Total RNA of the hMBs treated with $30 \mu \mathrm{M}$ iSN04 in DM for $24 \mathrm{~h}$ was subjected to RNAseq (Supplementary Figure 4A). 51.3 million reads per sample were acquired, of which approximately 45.9 million reads were mapped to a human reference genome (Supplementary Table 3). In total, 60,448 transcripts were identified and their expression levels were calculated as FPKM. FPKM values of myogenic genes exhibited a pattern compatible with qPCR results; iSN04 


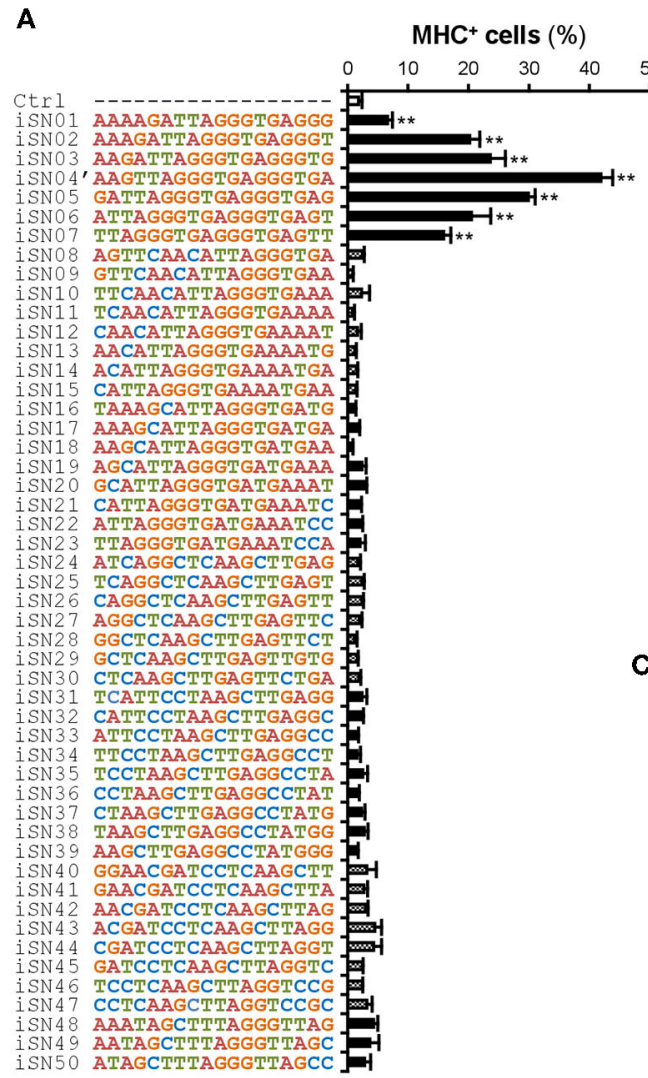

B
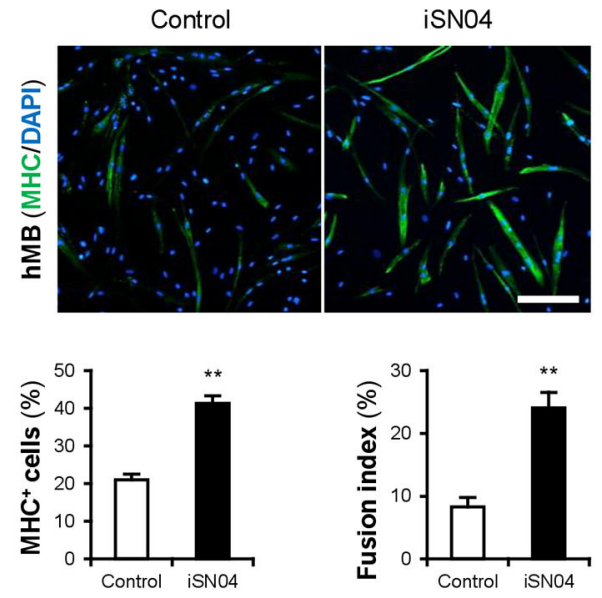

C
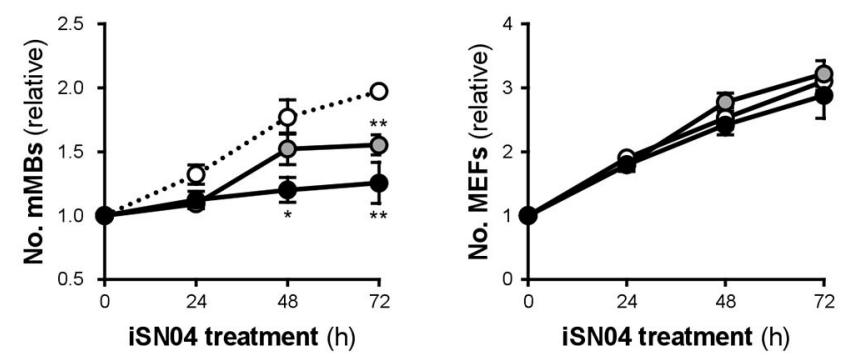

D
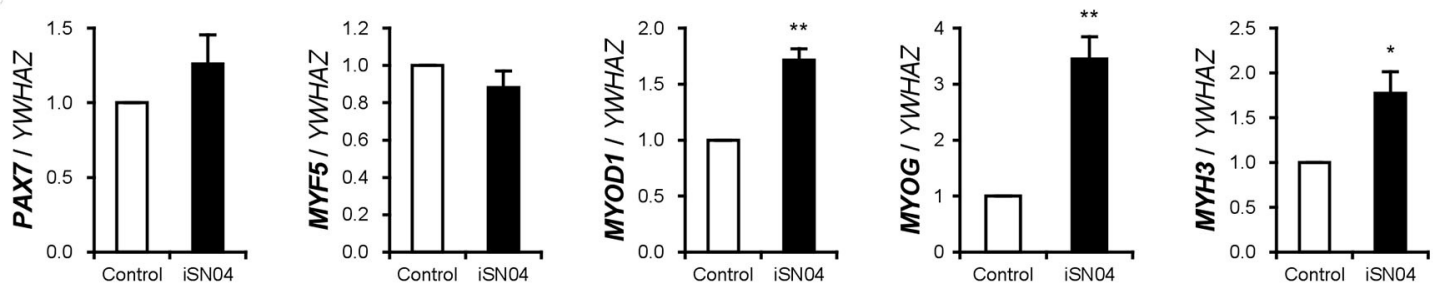

E

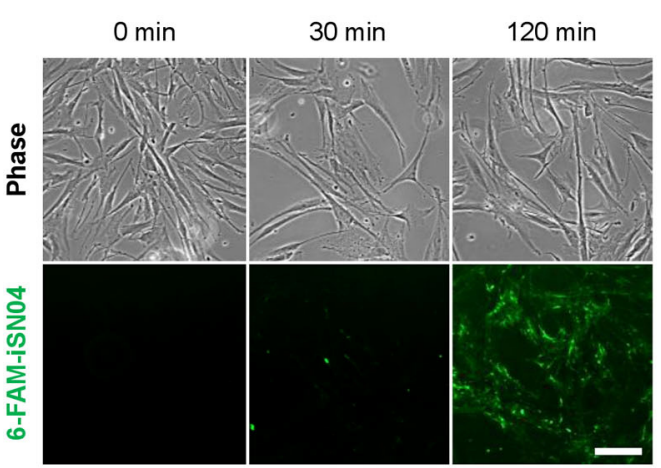

$\mathbf{F}$

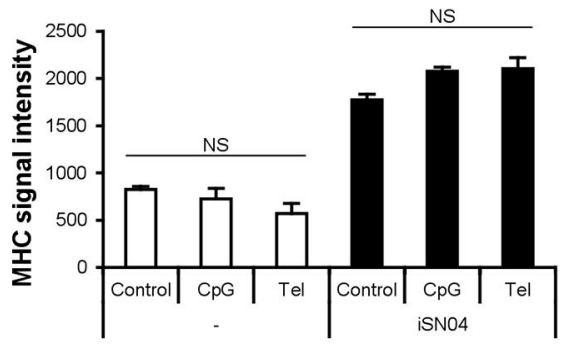

FIGURE 1 | Identification of myoDNs. (A) Ratio of $\mathrm{MHC}^{+}$cells within the screened mMBs treated with $10 \mu \mathrm{M}$ PS-ODNs in GM for $48 \mathrm{~h}$ (screening system). ${ }^{* *} p<0.01$ (Dunnett's test). $n=3$. (B) Representative immunofluorescent images of the hMBs treated with $10 \mu \mathrm{M}$ iSN04 in DM for $48 \mathrm{~h}$. Scale bar, $200 \mu \mathrm{m}$. Ratio of MHC ${ }^{+}$cells 
FIGURE 1 | and multinuclear myotubes were quantified. ${ }^{*} p<0.01$ (Student's $t$-test). $n=6$. (C) Relative numbers of the mMBs and MEFs treated with 1 or $3 \mu \mathrm{M}$ iSN04 in GM for each cell. Mean value of the control sample at $0 \mathrm{~h}$ was set to 1.0 for each experiment. ${ }^{*} p<0.05$, ${ }^{* *} p<0.01$ vs. control at each time point (William's test). $n=3$. (D) qPCR results of myogenic gene expression in the hMBs treated with $30 \mu \mathrm{M}$ iSN04 in DM for $24 \mathrm{~h}$. Mean value of control hMBs was set to $1.0 .{ }^{*} p<$ $0.05,{ }^{* *} p<0.01$ vs. control (Student's $t$-test). $n=3$. (E) Representative fluorescent images of the hMBs treated with $5 \mu \mathrm{g} / \mathrm{ml} 6$-FAM-iSN04 in GM. Scale bar, $100 \mu \mathrm{m}$. (F) MHC signal intensities of the hMBs treated with $30 \mu \mathrm{M}$ of iSN04, CpG-2006, or Tel-ODN in DM for 48h (screening system). NS, no significant difference (Scheffe's F-test). $n=3$.

significantly downregulated MYF5 and upregulated MYOD1 and MYOG (Supplementary Figure 4B). A total of 22,269 transcripts showed significant expression levels (FPKM >0.1) in the control or iSN04 group. Of them, 899 transcripts were differentially expressed ( $>1.5$-fold) with the significance of FDR $p<0.05$ (Supplementary Data). Of which 476 and 423 transcripts were upregulated and downregulated by iSN04, respectively. These DEGs depending on iSN04 were subjected to GO analysis. The 476 iSN04-upregulated DEGs significantly formed multiple gene clusters for muscle adaptation, contraction, and formation, which abundantly included sarcomeric components (myosin, actin, troponin, and their associated proteins) and transcription factors (myogenin, Hes1, Smad7, and Wnt10a) (Figure 2A). In contrast, the 423 iSN04-downregulated DEGs involved many clusters related to cell cycle and proliferation with higher significance (Figure 2B). These expression profiles of the iSN04-dependent DEGs corresponded well with the phenotype of iSN04-treated myoblasts, which showed promoted myogenic differentiation and arrested cell growth. STRING analysis visualized functional and physiological interactions of the DEGs or their products (Figure 2C). The tightly connected networks were detected in both DEG groups. Especially within the iSN04-downregulated group, 173 of the 423 DEGs (40.9\%) were concentrated in the primary cluster, suggesting that iSN04 possibly suppresses at least one of the major nodes of the transcriptome at the early stage regulating myoblast fate. These data indicate that iSN04 globally modulates gene expression by orchestrating the myogenic program and cell cycle in myoblasts.

\section{myoDN Activity Is Dependent on Its Structure}

ODNs are classified into three categories according to their mechanism of action: antisense nucleotides, aptamers, and immunogenic ODNs as TLR ligands. myoDN activity was independent of TLR signals. Furthermore, immunogenic ODNs are often species-specific (Pohar et al., 2015), but iSN04 induces the differentiation of both murine and human myoblasts. To investigate the potential of myoDNs as antisense ODNs, the homologous sequences of iSN04 in human and murine genomes were surveyed using BLAST. The BLAST results displayed 59 loci in humans and 39 loci in mice that had iSN04homologous sequences. However, there was no common gene or locus between humans and mice, denying that iSN04 serves as an antisense nucleotide. Intriguingly, the heat-denatured iSN02 lost the ability to induce myoblast differentiation (Supplementary Figure 2F), which strongly suggests that myoDN activity arises from its structure. Notably, iSN04 was resistant to thermal denaturation (Supplementary Figure 2G). The iSN04 conformation is considered to be relatively stable and can recover from denaturation in a short period. This might also be the reason why iSN04 presented the highest activity among the myoDNs.

The conformational properties of iSN04 under water conditions were computationally investigated using TTPMcMD (Ikebe et al., 2011). iSN04 at $310 \mathrm{~K}$ showed a compact globular structure (average radius: $0.96 \mathrm{~nm}$ ), not a linear strand (Figure 3A). iSN04 displayed varied conformations, but their variations seemed to be limited within a certain range (Supplementary Movie). For fine conformation analysis, the contact probabilities between the residues of iSN04 were calculated. The ensemble-averaged contact probabilities at $310 \mathrm{~K}$ over all the simulated iSN04 structures were rendered as a contact map (Figure 3B). Three guanines at the 13-15th bases stacked upon each other, suggesting that this $\mathrm{G}_{13-15}$ stack is the stable center of the iSN04 structure. The impact of the $\mathrm{G}_{13-15}$ bases on iSN04 activity was examined using mutant iSN04. A series of deletions in the $\mathrm{G}_{13-15}$ bases gradually attenuated the myogenetic activity of iSN04. In particular, iSN04 ${ }^{\Delta 13-15}$ completely lost its activity (Figure 3G), demonstrating that the $\mathrm{G}_{13-15}$ stack is indispensable for iSN04 activity.

\section{Berberine Enhances iSNO4 Activity}

We hypothesized that stabilization of the $\mathrm{G}_{13-15}$ stack would improve iSN04 activity. Telomeric DNA is known to form a highly ordered conformation. The G-quartet is a square aromatic surface constructed by the four guanines interacting with its neighbors via two hydrogen bonds. G-quartets stack upon each other to build the four-stranded G-quadruplex (Ou et al., 2008). Berberine, an isoquinoline alkaloid (Supplementary Figure 5A), interacts with the G-quartet and stabilizes the G-quadruplex structure derived from human telomeric DNA (Bazzicalupi et al., 2012). We tested whether iSN04 physically interacts with berberine. Briefly, iSN04 and berberine were mixed in F10 medium (Supplementary Table 4), subjected to electrophoresis, and stained with EtBr, which fluoresces red at $620 \mathrm{~nm}$. The iSN04-berberine complex can be imaged with yellow fluorescence because berberine fluoresces green at $530 \mathrm{~nm}$ (Guo et al., 2015; Shinji et al., 2020a). Indeed, yellow emissions of the iSN04-berberine complex were detected at a slightly higher molecular weight compared to iSN04 alone (Figure 3E). Not only iSN04 but also all other myoDNs interacted with berberine (Supplementary Figure 5C). G-quartets generally coordinate cations (Ou et al., 2008), and berberine binds to the telomeric DNA holding $\mathrm{K}^{+}$(Bazzicalupi et al., 2012). We examined the requirement of cations for the iSN04-berberine complex using cationic solutions. $\mathrm{Ca}^{2+}$ was found to be necessary for the iSN04-berberine complex (Supplementary Figure 5B). Moreover, $\mathrm{Mg}^{2+}$ facilitated the interaction between iSN04 and 
A

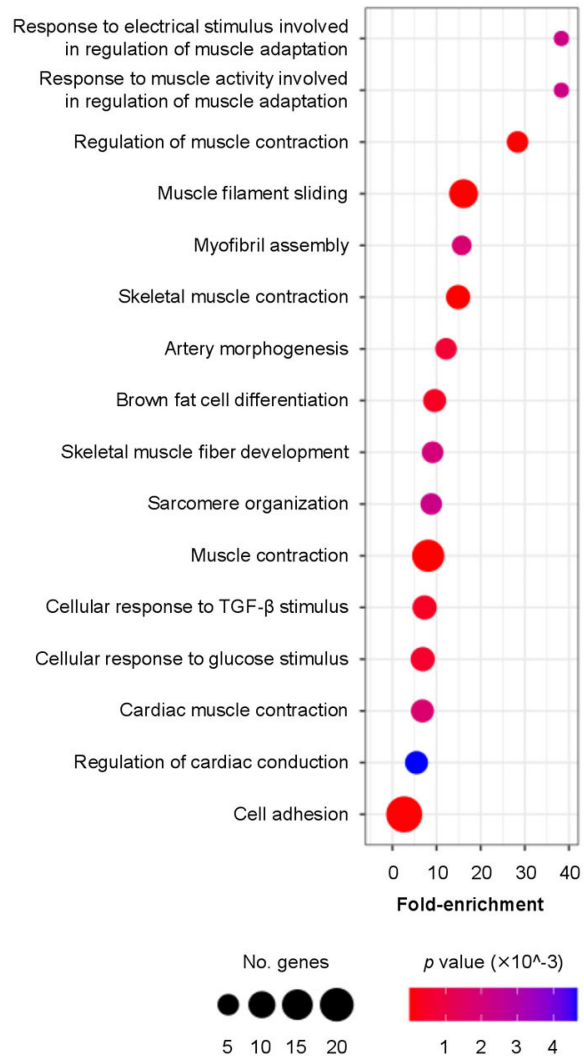

C

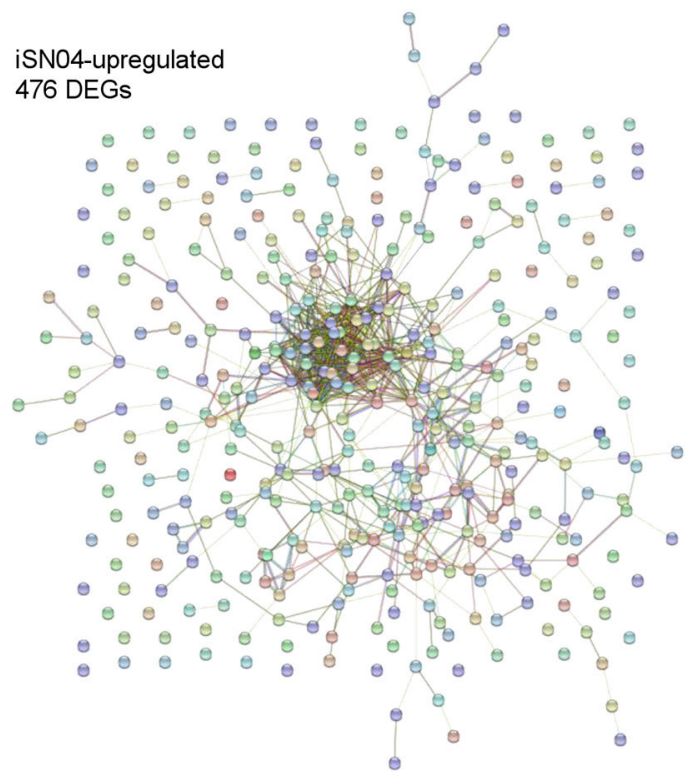

B

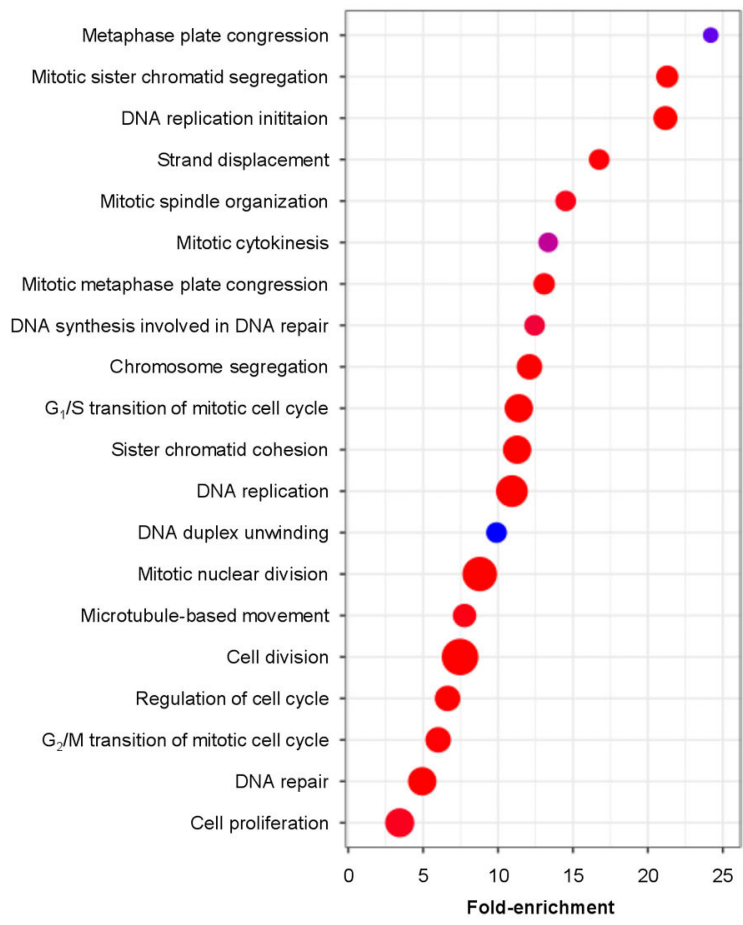

No. genes $\quad p$ value $\left(\times 10^{\wedge}-6\right)$

$\bullet \bullet \bullet ० 0$

$10 \quad 20 \quad 30 \quad 40 \quad 50$

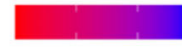

2

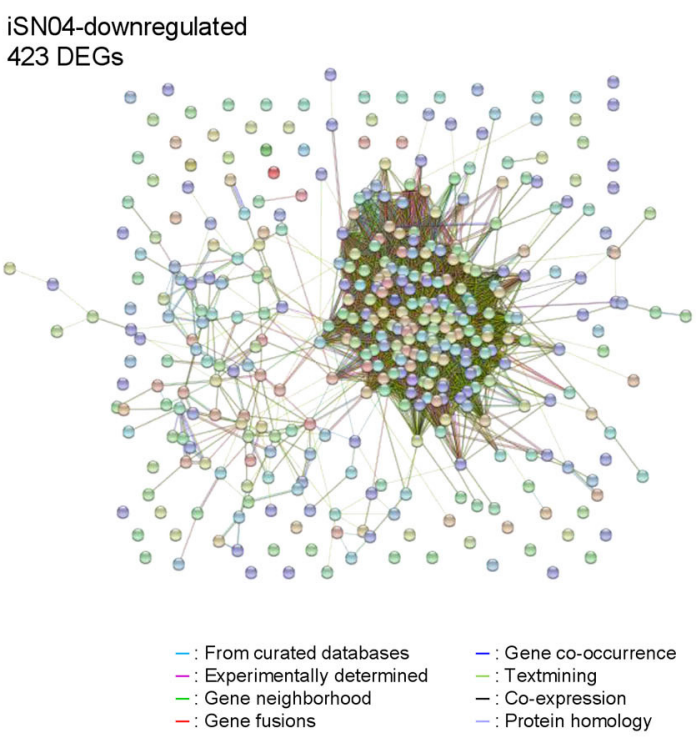

FIGURE 2 | Profile of iSN04-dependent gene expression. (A) Scattered plot of the 476 iSN04-upregulated DEGs significantly $\left(p<5.0 \times 10^{-3}\right)$ enriched in GO terms. (B) Scattered plot of the 423 iSN04-downregulated DEGs significantly $\left(p<5.0 \times 10^{-6}\right)$ enriched in GO terms. (C) Functional and physiological networks within the 476 iSN04-upregulated DEGs (left panel) and the 423 iSN04-downregulated DEGs (right panel) visualized via STRING analysis. 
A

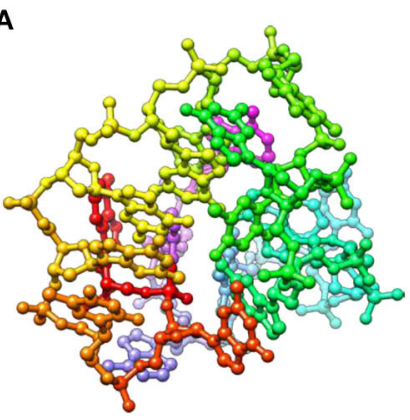

B

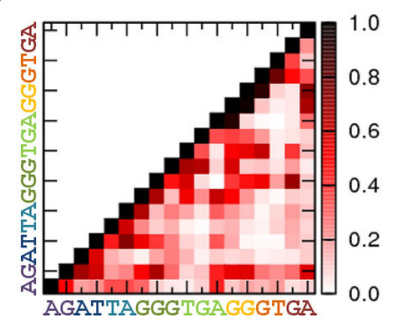

C

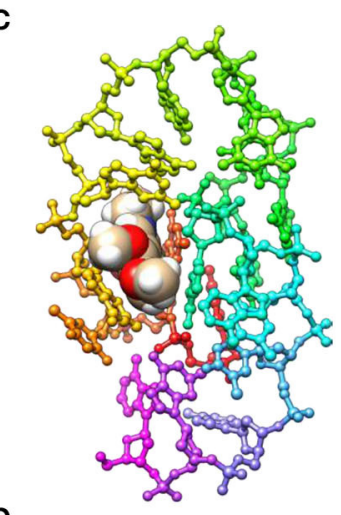

D

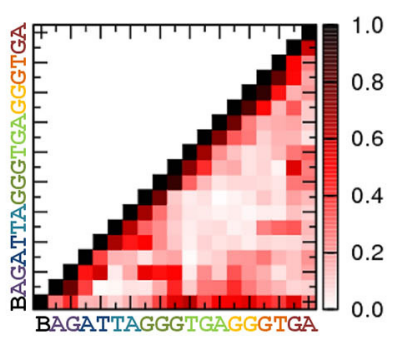

E

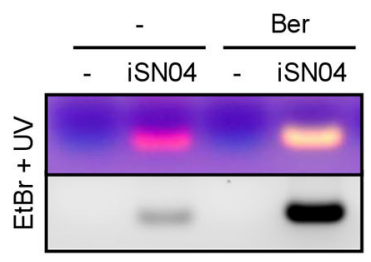

$\mathbf{F}$

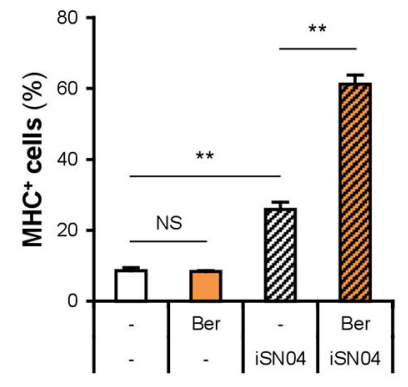

G

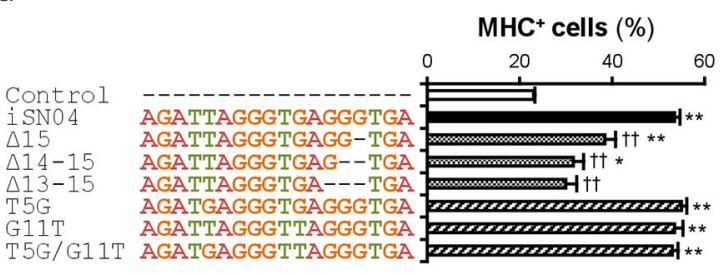

H

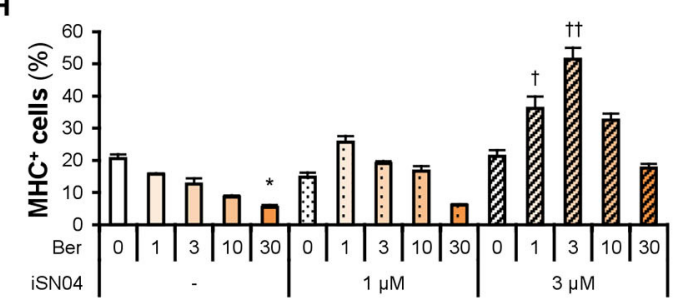

I

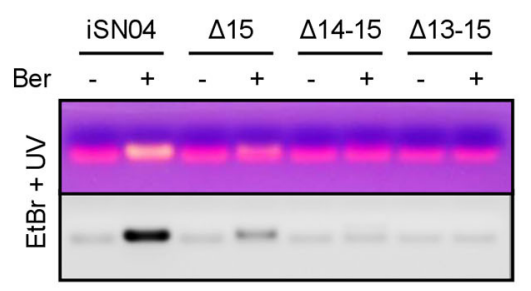

$\frac{\text { iSN04 }}{+} \frac{\text { T5G }}{++} \frac{\text { G11T }}{+} \frac{\text { T5G/G11T }}{+}$

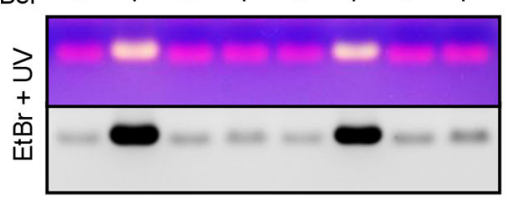

J

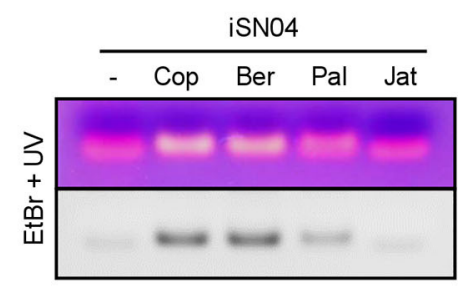

$\mathbf{K}$

口iSNO4(-) घiSNO4(+)

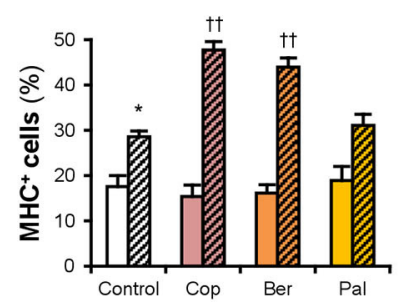

FIGURE 3 | Berberine enhances iSN04 activity by causing a shift in molecular structure. (A) The conformation of iSN04 simulated via TTP-McMD. (B) Contact map of iSN04. The scale indicates contact probability. (C) The simulated conformation of iSN04-berberine complex. Berberine is shown as a sphere model. (D) Contact map 
FIGURE 3 | of iSN04-berberine complex. "B" indicates berberine. (E) Representative images of agarose gel electrophoresis of iSN04 mixed with berberine (Ber) in F10 medium. The EtBr-stained and UV-irradiated gel was scanned without filters (upper image) and with a 560-nm filter (lower image). (F) Ratio of MHC ${ }^{+}$cells within the mMBs treated with $10 \mu \mathrm{M}$ iSN04 and $10 \mu \mathrm{M}$ berberine in GM for $48 \mathrm{~h}$ (screening system). ${ }^{\star \star} p<0.01$; NS, no significant difference (Scheffe's $F$-test). $n=3$. (G) Ratio of $\mathrm{MHC}^{+}$cells within the mMBs treated with $10 \mu \mathrm{M}$ of mutant iSN04 in GM for $48 \mathrm{~h}$ (screening system). ${ }^{*} p<0.05,{ }^{* *} p<0.01$ vs. control; ${ }^{+\dagger} p<0.01$ vs. iSN04 (Scheffe's F-test). $n=3$. (H) Ratio of $\mathrm{MHC}^{+}$cells within the mMBs treated with 0 , 1, or $3 \mu \mathrm{M}$ iSN04 and 0, 1, 3, 10 , or $30 \mu \mathrm{M}$ of berberine in GM for $48 \mathrm{~h}$ (screening system). ${ }^{*} p<0.05$ vs. $0 \mu \mathrm{M}$-iSN04 $+0 \mu \mathrm{M}$-berberine; ${ }^{\dagger} p<0.05,{ }^{\dagger \dagger} p<0.01$ vs. $3 \mu \mathrm{M}$-iSN04 $+0 \mu \mathrm{M}$-berberine (Scheffe's $F$-test). $n=3$. (I) Representative images of agarose gel electrophoresis of mutant iSN04 mixed with berberine in F10 medium. (J) Representative images of agarose gel electrophoresis of iSN04 mixed with coptisine (Cop), berberine, palmatine (Pal), or jatrorrhizine (Jat) in F10 medium. (K) Ratio of $\mathrm{MHC}^{+}$cells within the mMBs treated with $10 \mu \mathrm{M}$ iSNO4 and $10 \mu \mathrm{M}$ berberine analogs in GM for $48 \mathrm{~h}$ (screening system). ${ }^{*} p<0.05$ vs. control-iSN04(-); ${ }^{\dagger \dagger} p<0.01$ vs. control-iSN04(+) (Tukey-Kramer test). $n=3$.

berberine, but its effect was markedly weaker than that of $\mathrm{Ca}^{2+}$. These results suggest that berberine binds to the G-quartet- or G-quadruplex-like structure within iSN04, which is probably formed by the $\mathrm{G}_{13-15}$ stack.

Administration of iSN04 and berberine to mMBs proved that the activity of the iSN04-berberine complex was significantly higher than that of single iSN04 (Figure 3F). As berberine alone did not alter myoblast differentiation, it is possible that the improved activity of the iSN04-berberine complex is not a synergistic effect. Berberine is speculated to enhance the inherent activity of iSN04 by stabilizing or shifting the conformation. In some cases, one G-quartet binds to two berberine molecules (Bazzicalupi et al., 2012). To optimize the molar ratio of iSN04 to berberine, mMBs were treated with $0-3 \mu \mathrm{M}$ iSN04 and 0-30 $\mu \mathrm{M}$ berberine. iSN04 exhibited the highest myogenetic activity when $\mathrm{mMBs}$ were co-treated with an equal molar of berberine (Figure 3H). Conformation of the iSN04-berberine complex at a molar ratio of 1:1 was simulated using TTPMcMD. Berberine interacted exactly with the $\mathrm{G}_{13-15}$ stack of iSN04 (Figure 3C). Deletions of the $\mathrm{G}_{13-15}$ bases of iSN04 experimentally demonstrated that berberine actually interacts with these guanines (Figure 3I, upper panel). The contact map of the iSN04-berberine complex showed that the $\mathrm{G}_{7-9}$ bases are stacked in addition to the $\mathrm{G}_{13-15}$ stack (Figure 3D). Berberine also contacted the $\mathrm{G}_{9}$ and consequently, it fits into the pocket assembled from the $G_{7-9}$ and $G_{13-15}$ stacks. iSN04 has two telomeric hexamers; TTAGGG and TGAGGG. We investigated the influence of the $\mathrm{T}_{5}$ and $\mathrm{G}_{11}$ of iSN04 on myogenetic activity and berberine binding. Both the T5G and G11T substitutions did not affect iSN04 activity (Figure 3G). However, the T5G substitution interfered with the formation of iSN04-berberine complex, and iSN04 ${ }^{\mathrm{G} 11 \mathrm{~T}}$ interacted with berberine as well as intact iSN04 (Figure 3I, lower panel). The contact maps indicated that the $T_{5}$ relatively remains at a distance from other bases. On the contrary, guanines tend to interact with other bases. The extra guanine inserted by the T5G substitution might have perturbed the pocket required for iSN04 to bind to berberine.

We further examined the iSN04-enhancing abilities of three berberine analogs, coptisine, palmatine, and jatrorrhizine (Supplementary Figure 5A). Coptisine and berberine formed a complex with iSN04. Palmatine weakly interacted with iSN04, but jatrorrhizine did not interact at all (Figure 3J). Correspondingly, coptisine significantly improved the myogenetic activity of iSN04 to the same level as the iSN04berberine complex (Figure $\mathbf{3 K}$ ). These results illustrate that the 2,3-methylenedioxy ring of the berberine backbone is important for interacting with iSN04.

\section{iSN04 Targets Nucleolin and Increases p53 Protein}

The structure-dependent myogenetic activity of iSN04 suggests the presence of iSN04-target proteins. We surveyed iSN04binding proteins by precipitation assay. Biotin-conjugated iSN04 was immobilized on streptavidin-beads at the $5^{\prime}$ or $3^{\prime}$ end (iSN04-5'-Bio and iSN04-3'-Bio, respectively). Soluble wholecell lysates were pre-pulled-down with iSN14-beads to eliminate the absorption of non-specific proteins onto ODNs or beads. After removing off-target proteins, the lysates were precipitated with iSN04-beads, followed to SDS-PAGE and CBB staining. Surprisingly, iSN04-binding protein was not detected in C2C12 cell lysates (data not shown). Next, the lysate of the murine osteoblast cell line MC3T3-E1 was prepared because iSN04 affected MC3T3-E1 differentiation (unpublished data). Both iSN04-5' -Bio and iSN04-3'-Bio precipitated the identical single protein with an expected size of $112 \mathrm{kDa}$, which was not precipitated by iSN14-beads or beads alone (Figure 4A). Mass spectrometry identified the iSN04-binding protein as nucleolin (Supplementary Tables 5, 6). Although the molecular weight of nucleolin is $77 \mathrm{kDa}$, it is practically detected at $100-110$ $\mathrm{kDa}$ because of the acidic amino acids in the $\mathrm{N}$-terminal domain (Jia et al., 2017). Nucleolin is a multifunctional phosphoprotein located in the nucleolus, cytoplasm, and plasma membrane depending on the context of cellular processes such as gene expression, protein shuttling, cytokinesis, and apoptosis. Expression and subcellular localization of nucleolin are frequently abnormal in rapidly growing cells, typically cancers (Jia et al., 2017). A recent study reported that the amount and localization of nucleolin is involved in myogenic differentiation of C2C12 cells (Tang et al., 2017), but its precise mechanism is still unknown.

A 26-base G-rich non-immunogenic ODN, AS1411 (Supplementary Table 1), is an established anti-nucleolin aptamer which has been utilized in several clinical trials on cancers (Bates et al., 2009; Yazdian-Robati et al., 2019). AS1411 promoted myogenic differentiation of hMBs to the same extent as iSN04 (Figure 4B), proving that iSN04 targets nucleolin in myoblasts. Immunostaining revealed that nucleolin initially localized in the nucleoli of hMBs and $\mathrm{C} 2 \mathrm{C} 12$ cells in their undifferentiated states. Nucleolin was then translocated into the cytoplasm of $\mathrm{MHC}^{+}$myotubes throughout myogenic differentiation (Figure 4C and Supplementary Figure 6A) 
A

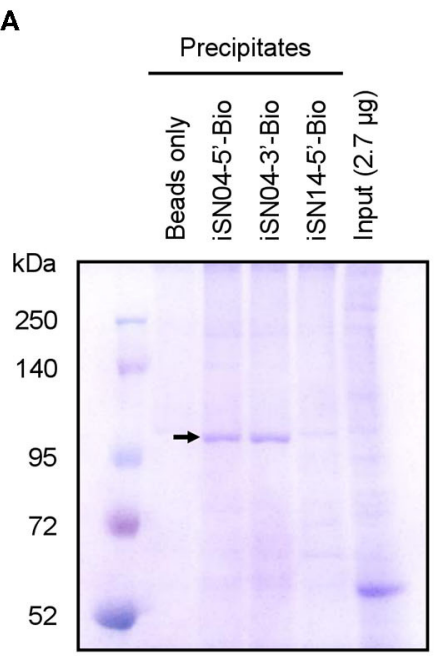

B
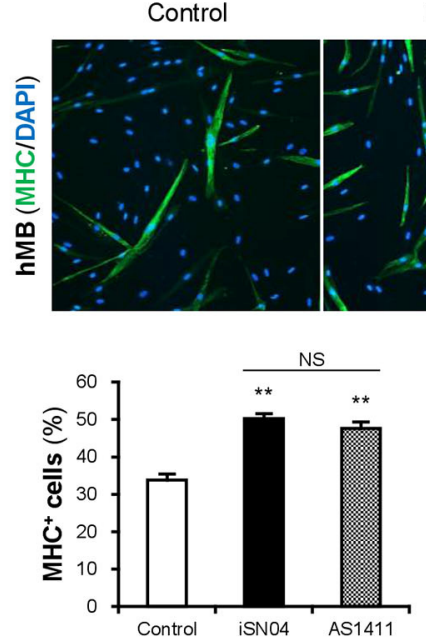

iSNO4
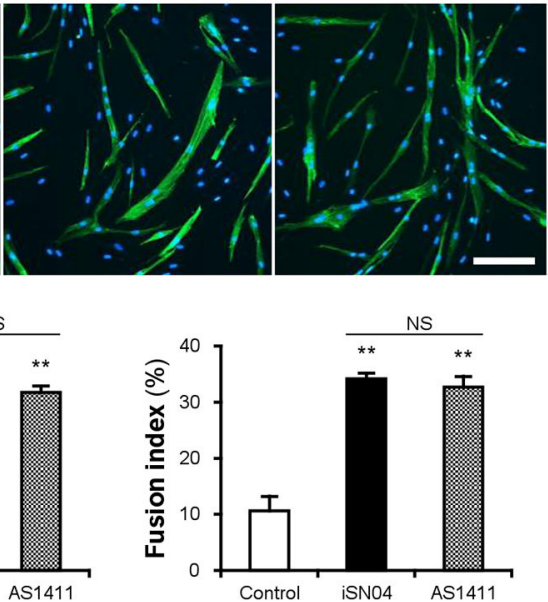

C

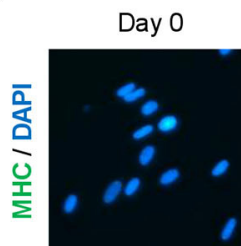

Day 4

D

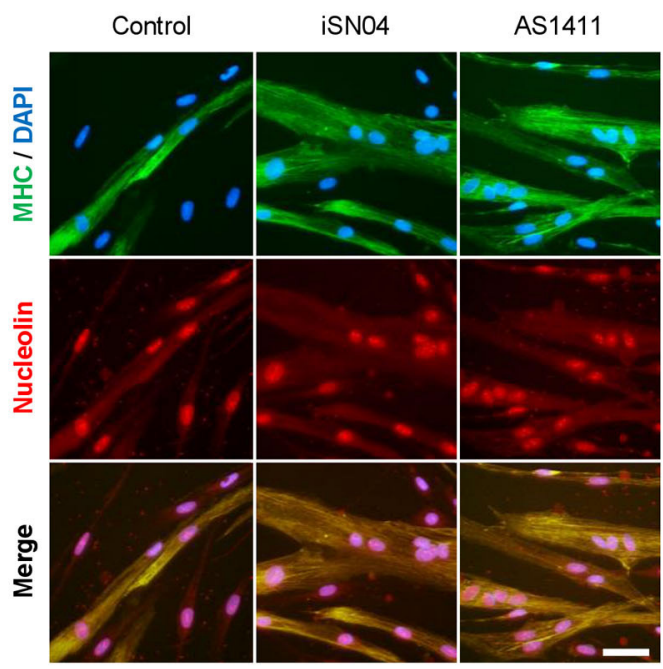

$\mathbf{E}$

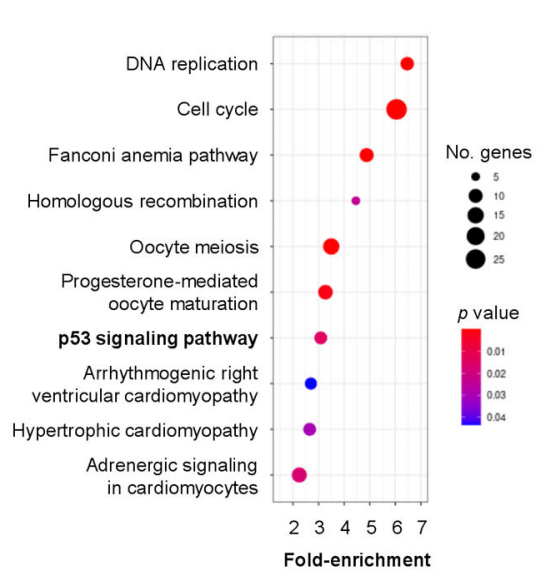

$\mathbf{F}$

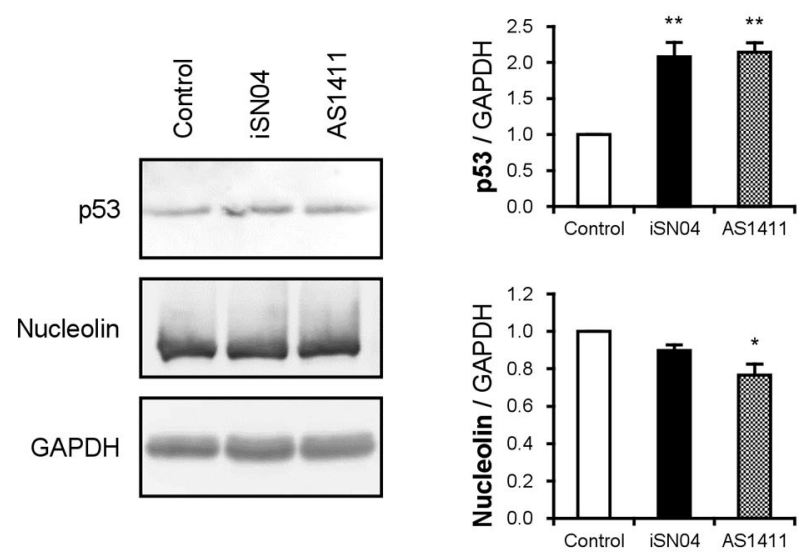

FIGURE 4 | iSN04 targets nucleolin and improves p53 protein level. (A) Representative image of CBB-stained SDS-PAGE gel of the ODN-precipitated proteins. The arrow indicates the bands subjected to mass spectrometry. (B) Representative immunofluorescent images of the hMBs treated with $10 \mu \mathrm{M}$ of iSN04 or AS1411 in DM (Continued) 
FIGURE 4 | for $48 \mathrm{~h}$. Scale bar, $200 \mu \mathrm{m}$. Ratio of $\mathrm{MHC}^{+}$cells and multinuclear myotubes were quantified. ${ }^{* \star} p<0.01 \mathrm{vs}$. control; NS, no significant difference (Student's t-test). $n=6$. (C) Representative immunofluorescent images of the hMBs maintained in DM at day 0, 2, and 4. Scale bar, $50 \mu \mathrm{m}$. (D) Representative immunofluorescent images of the hMBs treated with $30 \mu \mathrm{M}$ of iSN04 or AS1411 in DM for $48 \mathrm{~h}$. Scale bar, $50 \mu \mathrm{m}$. (E) Scattered plot of the 899 iSN04-dependent DEGs significantly (FDR $p<0.05$ ) enriched in KEGG pathways. (F) Representative images of Western blotting (10 $\mu \mathrm{g}$ protein/lane) of p53, nucleolin, and GAPDH in soluble whole cell lysates of the hMBs treated with $30 \mu \mathrm{M}$ of iSN04 or AS1411 in DM for $48 \mathrm{~h}$. Amounts of p53 and nucleolin were normalized using GAPDH. Mean value of control hMBs was set to 1.0. ${ }^{\star} p<0.05,{ }^{\star \star} p<0.01$ vs. control (Scheffe's $F$-test). $n=3$.

as previously reported (Tang et al., 2017). The mRNA levels of nucleolin (NCL) in hMBs were increased during differentiation but did not change after iSN04 treatment (Supplementary Figures 6C,D). In addition, nucleolin localization in hMBs or C2C12 cells was not shifted by iSN04 or AS1411 (Figure 4D and Supplementary Figure 6B). These results correspond to those of previous studies, which confirmed that AS1411 does not alter nucleolin localization in cancer cells (Litchfield et al., 2012; Reyes-Reyes et al., 2015; Ramos et al., 2020). Nucleolin has been reported to target the untranslated region (UTR) of p53 mRNA to interfere with its translation in tumor cells (Takagi et al., 2005; Chen et al., 2012). Nucleolin inhibition by AS1411 increases p53 protein levels and suppresses cell proliferation in glioma cells (Cheng et al., 2016). As p53 induces myogenic differentiation (Cerone et al., 2000; Porrello et al., 2000), antagonizing nucleolin in myoblasts by iSN04 or AS1411 was assumed to upregulate p53 protein and promote differentiation. RNA-seq data showed that the iSN04-dependent DEGs were enriched in the p53 signaling pathway (Figure 4E and Supplementary Figure 6E). Western blotting showed that both iSN04 and AS1411 significantly increased p53 protein levels in hMBs (Figure 4F), even though iSN04 decreased the mRNA level of p53 (TP53) (Supplementary Figure 6F).

These data demonstrate that iSN04 antagonizes nucleolin to recover the suppressed p53 translation. The iSN04-increased p53 protein level activates downstream signal to arrest the cell cycle and induce myoblast differentiation. The results of this study present evidence that bacterial-derived ODNs can serve as aptamers to modulate cell fate.

\section{DISCUSSION}

To our knowledge, this is the first report of the ODNs promoting myogenic differentiation of skeletal muscle myoblasts. myoDNs are 18-base telomeric ODNs designed from the LGG genome sequence. Such bacterial ODNs often serve as immunogenic ODNs recognized by TLRs and modulate the innate immune system (Krieg et al., 1995; Klinman et al., 2008). Among them, telomeric ODNs, also termed iODNs, are known to suppress inflammatory responses (Sackesen et al., 2013; Wang Y. et al., 2015). In our previous study, myoDNs (iSN01-iSN07) were not iODNs (Nigar et al., 2017). iSN04, the myoDN presenting the highest activity, induced myoblast differentiation independent of TLR signaling. myoDNs are thus defined as a novel type of ODN that regulates cell fate through a unique mechanism. A previous study reported that CpG-2006 interferes with the osteoblastic differentiation of MSCs in a TLR9-independent manner, but its direct target is unknown (Norgaard et al., 2010). CpG-2006 was originally identified as a TLR9 ligand that activates immune responses (Hartmann et al., 2000; Bauer et al., 2001). The dual role of CpG-2006, in addition to myoDNs, implies that other bacterial ODNs might also exert non-immunological functions.

The present study revealed that the myogenetic activity of iSN04 arises from its conformation rather than its sequence. Molecular simulation and a series of mutant iSN04 demonstrated that the $\mathrm{G}_{13-15}$ stack within the second telomeric hexamer is essential for iSN04 activity. It is also indicated that berberine physically interacts with iSN04 via the $\mathrm{G}_{13-15}$ stack and enhances the myogenetic ability of iSN04, probably by stabilizing or optimizing the structure. This is the initial instance of functional improvement of ODNs by small molecules. Berberine is a safe isoquinoline alkaloid isolated from medicinal plants, that exhibits various bioactivities and has been utilized in clinical studies on diabetes, osteoporosis, and cancer (Imenshahidi and Hosseinzadeh, 2019). Berberine has also been studied as a ligand of the G-quadruplex, which is often formed in oncogenic promoter regions and regulates gene expression by recruiting transcriptional machinery (Siddiqui-Jain et al., 2002). Therefore, berberine derivatives that block G-quadruplexes can be potential anti-tumor drugs (Ou et al., 2008; Bazzicalupi et al., 2012). From these points of view, the modification of the structure and function of telomeric ODN by berberine provides a safe and reasonable technique for further research, development, and application of ODNs.

We identified nucleolin as a direct target of iSN04. The established anti-nucleolin aptamer, AS1411, also promoted myoblast differentiation, which proved that iSN04 antagonizes nucleolin. AS1411 has been reported to polymorphically fold into various conformations, including G-quadruplex structures (Dailey et al., 2010; Supplementary Figure 6G). Interestingly, iSN04 but not AS1411 interacted with berberine (Supplementary Figure 5D), and AS1411 but not iSN04 decreased nucleolin levels (Figure 4F), which suggests the presence of subtle structural and functional differences. Experimental determination of the iSN04 structure will provide valuable information on the similarity and dissimilarity between iSN04 and AS1411, which will be useful for building novel aptamers based on their conformations. Aptamers are usually developed via in vitro methodology using a specific target, systemic evolution of ligands by exponential enrichment (SELEX) (Wang et al., 2019). However, AS1411 is a non-SELEX aptamer that was screened as an inhibitor for cancer cell growth (Bates et al., 2009). Precipitation assay and mass spectrometry identified nucleolin as an AS1411 target (Girvan et al., 2006). Although AS1411 interacts with at least 15 proteins including nucleolin (Girvan et al., 2006), nucleolin has been a primary target of AS1411. Because aptamers usually recognize membrane proteins, and nucleolin is present on the plasma membrane of 
cancer cells (Bates et al., 2009; Jia et al., 2017; Yazdian-Robati et al., 2019).

Unlike many aptamers, iSN04 and AS1411 exert their effects inside myoblasts. iSN04 was spontaneously incorporated into myoblasts. Nucleolin initially localized in the nucleoli of growing myoblasts and diffused into the cytoplasm during myotube formation. However, iSN04 and nucleolin were not observed on the surface of myoblasts throughout differentiation. As discussed below, nuclear nucleolin serves as an mRNAbinding protein that regulates translation (Fahling et al., 2006). These findings indicate that iSN04 and AS1411 conceivably function in the nuclei of myoblasts. In general, singlestrand ODNs are efficiently taken into the cytoplasm without carriers through gymnosis. Although its mechanism has not been completely understood, ODNs are considered to be incorporated by endocytosis, transported to the endosome, and are transferred to the cytoplasm through the endosomal membrane, probably due to their lower molecular weights and higher hydrophobicities compared to double-strand nucleotides. The released ODNs into the cytoplasm can accumulate in the nucleus by associating with chaperones or RNA-binding proteins (Juliano, 2018).

Nucleolin interferes with the translation of p53 mRNA by binding to its UTR (Takagi et al., 2005; Chen et al., 2012). Our study showed that antagonizing nucleolin by iSN04 or AS1411 increased p53 protein levels in myoblasts, as reported in AS1411-treated glioma cells (Cheng et al., 2016). The role of p53 in myoblasts has been intensively studied. An initial study found that the dominant-negative form of p53 inhibits the differentiation of C2C12 cells (Soddu et al., 1996). During myogenic differentiation, p53 cooperates with MyoD (Cerone et al., 2000) to activate transcription of retinoblastoma protein (Porrello et al., 2000), which serves as a cofactor of MyoD to arrest the cell cycle and facilitate muscle cell commitment (Gu et al., 1993; Novitch et al., 1996). A recent study revealed that p53 with MyoD coactivates the expression of the pro-apoptotic protein PUMA (Harford et al., 2017), which is required for the apoptosis associated with myoblast differentiation (Shaltouki et al., 2007; Harford et al., 2010). This accumulating evidence corroborates the findings that iSN04 upregulates $\mathrm{p} 53$ protein and induces myoblast differentiation.

Interestingly, iSN04 did not affect the growth of MEFs expressing nucleolin (Supplementary Figure 6H). In the precipitation assays, iSN04 pulled down nucleolin in the lysates of MC3T3-E1 cells but not of $\mathrm{C} 2 \mathrm{C} 12$ cells, even though the amounts of nucleolin were nearly equal between the lysates (Supplementary Figure 6I). According to circumstances, nucleolin is post-translationally modified such as phosphorylation and glycosylation (Barel et al., 2001; Losfeld et al., 2009), and interacts with various partners including nucleotides and proteins (Jia et al., 2017). Probably due to that, AS1411 precipitates only certain forms of nucleolin (Teng et al., 2007; Bates et al., 2009). The amounts of the iSN04-binding form of nucleolin in $\mathrm{C} 2 \mathrm{C} 12$ cells might be less than that in MC3T3-E1 cells and not enough to be detected via CBB staining. It is possible that the mode of existence of nucleolin differs among the cells, which affects its affinity to iSN04.
The precise role of nucleolin during myogenic differentiation is still not fully understood. A moderate decline in nucleolin protein by miR-34b has been reported to upregulate myogenic expression (Tang et al., 2017). This study showed that nucleolin levels decreased through differentiation of C2C12 cells; however, our results using primary-cultured hMBs showed increased nucleolin expression upon differentiation. As nucleolin is potently induced in actively proliferating cells like tumors (Jia et al., 2017), nucleolin levels might be high in the immortalized $\mathrm{C} 2 \mathrm{C} 12$ cell line. Therefore, nucleolin function in myoblasts needs to be further investigated using primarycultured cells or in vivo models. In both hMBs and C2C12 cells, nucleolin initially localized in the nucleoli and then diffused into the cytoplasm through differentiation. An analogous shift of nucleolin localization has been observed during adipogenic differentiation of 3T3-L1 pre-adipocytes (Wang H. et al., 2015). The biological activities of nucleolin can vary depending on its subcellular distribution. Numerous studies have revealed that nucleolar nucleolin regulates RNA metabolism, nucleoplasmic nucleolin modulates gene expression, cytoplasmic nucleolin serves as a shuttle protein, and cell surface nucleolin is involved in various signaling pathways (Jia et al., 2017). Elucidating the relationship between nucleolin localization and differentiation of precursor cells would be important to understand the fine-tuned mechanism of myoDNs.

To establish myoDNs as potential drug seeds for muscle diseases, their pharmacological actions need to be investigated in vivo. Intramuscular injection (i.m.) of antisense nucleotides have been clinically applied to treat Duchenne muscular dystrophy (Quemener et al., 2020). However, i.m. is painful and there is a risk of sterile abscess formation, therefore it is not suitable for prevention or treatment of the long-termed muscle atrophy associating with aging or chronic diseases. We have previously developed ODN nanocapsules (ODNcaps) as an oral delivery system of ODNs. Oral administration of the capsuled iODN to atopic model mice successfully suppressed immune responses in dermatitis (Wang Y. et al., 2015). ODNcaps would be also useful to deliver myoDNs to atrophic muscle tissue. Further studies using adequate animal model and drug delivery system are required for clinical application of myoDNs in future.

\section{CONCLUSION}

This study presents that bacterial genome-derived myoDNs promote myogenic differentiation by targeting nucleolin. The myoDN activities can be enhanced by conformational changes via binding to berberine. myoDNs are expected to be novel and unique drug candidates for muscle diseases, including atrophy, in which myoblasts are functionally deteriorated.

\section{DATA AVAILABILITY STATEMENT}

The datasets generated for this study can be found in online repositories. The names of the repository/repositories and accession number(s) can be found in the article/Supplementary Material. 


\section{ETHICS STATEMENT}

All experimental procedures were conducted in accordance with the Regulations for Animal Experimentation of Shinshu University, and the animal experimentation protocol was approved by the Committee for Animal Experiments of Shinshu University.

\section{AUTHOR CONTRIBUTIONS}

TT designed the study. TT and KU wrote the manuscript. SS, YN, $\mathrm{SN}$, and TT performed the experiments and data analyses. KU performed molecular simulation and proposed iSN04-berberine interaction. TS designed and provided the ODNs. All authors contributed to the article and approved the submitted version.

\section{FUNDING}

This study was supported in part by Grants-in Aid from the Japan Society for the Promotion of Science (16K19397 and

\section{REFERENCES}

Amcheslavsky, A., Hemmi, H., Akira, S., and Bar-Shavit, Z. (2005). Differential contribution of osteoclast- and osteoblast-lineage cells to CpG-oligodeoxynucleotide (CpG-ODN) modulation of osteoclastogenesis. J. Bone Miner. Res. 20, 1692-1699. doi: 10.1359/JBMR.050515

Anders, S., Pyl, P. T., and Huber, W. (2015). HTSeq - a python framework to work with high-throughput sequencing data. Bioinformatics 31, 166-169. doi: 10.1093/bioinformatics/btu638

Anker, S. D., Ponikowski, P., Varney, S., Chua, T. P., Clark, A. L., Webb-Peploe, K. M., et al. (1997). Wasting as independent risk factor for mortality in chronic heart failure. Lancet 349, 1050-1053. doi: 10.1016/S0140-6736(96)07015-8

Babicki, S., Arndt, D., Marcu, A., Liang, Y., Grant, J. R., Maciejewski, A., et al. (2016). Heatmapper: web-enabled heat mapping for all. Nucleic Acids Res. 44, W147-W153. doi: 10.1093/nar/gkw419

Barel, M., Le Romancer, M., and Frade, R. (2001). Activation of the EBV/C3d receptor (CR2, CD21) on human B lymphocyte surface triggers tyrosine phosphorylation of the $95-\mathrm{kDa}$ nucleolin and its interaction with phosphatidylinositol 3 kinase. J. Immunol. 166, 3167-3173. doi: 10.4049/jimmunol.166.5.3167

Bates, P. J., Laber, D. A., Miller, D. M., Thomas, S. D., and Trent, J. O. (2009). Discovery and development of the G-rich oligonucleotide AS1411 as a novel treatment for cancer. Exp. Mol. Pathol. 86, 151-164. doi: 10.1016/j.yexmp.2009.01.004

Bauer, S., Kirschning, C. J., Hacker, H., Redecke, V., Hausmann, S., Akira, S., et al. (2001). Human TLR9 confers responsiveness to bacterial DNA via speciesspecific CpG motif recognition. Proc. Natl. Acad. Sci. U.S.A. 98, 9237-9242. doi: 10.1073/pnas.161293498

Bazzicalupi, C., Ferraroni, M., Bilia, A. R., Scheggi, F., and Gratteri, P. (2012). The crystal structure of human telomeric DNA complexed with berberine: an interesting case of stacked ligand to G-tetrad ratio higher than 1:1. Nucleic Acids Res. 41, 632-638. doi: 10.1093/nar/gks1001

Brack, A. S., Conboy, M. J., Roy, S., Lee, M., Kuo, C. J., Keller, C., et al. (2007). Increased Wnt signaling during aging alters muscle stem cell fate and increases fibrosis. Science 317, 807-810. doi: 10.1126/science.1144090

Carrero, J. J., Chmielewski, M., Axelsson, J., Snaedal, S., Heimburger, O., Barany, P., et al. (2008). Muscle atrophy, inflammation and clinical outcome in incident and prevalent dialysis patients. Clin. Nutr. 27, 557-564. doi: 10.1016/j.clnu.2008.04.007

Cerone, M. A., Marchetti, A., Bossi, G., Blandino, G., Sacchi, A., and Soddu, S. (2000). p53 is involved in the differentiation but not in the
19K05948), the TOBE MAKI Scholarship Foundation (17-JA503), the Skylark Food Science Institute, the Takano Science Foundation, and the Japan Society for Bioscience, Biotechnology, and Agrochemistry to TT, and a Grant-in-Aid from the Fund of Nagano Prefecture to Promote Scientific Activity (H30-3-3) to SS.

\section{ACKNOWLEDGMENTS}

C57BL/6J mice and $\mathrm{C} 2 \mathrm{C} 12$ cells were kindly provided by Dr. Sachi Tanaka and Dr. Shinichi Yonekura, of Shinshu University. The preprint has been posted on bioRxiv (Shinji et al., 2020b).

\section{SUPPLEMENTARY MATERIAL}

The Supplementary Material for this article can be found online at: https://www.frontiersin.org/articles/10.3389/fcell.2020. 616706/full\#supplementary-material differentiation-associated apoptosis of myoblasts. Cell Death Differ. 7, 506-508. doi: 10.1038/sj.cdd.4400676

Chang, J. H., Chang, E. J., Kim, H. H., and Kim, S. K. (2009). Enhanced inhibitory effects of a novel CpG motif on osteoclast differentiation via TREM-2 down-regulation. Biochem. Biophys. Res. Commun. 389, 28-33. doi: $10.1016 /$ j.bbrc.2009.08.080

Chen, J., Guo, K., and Kastan, M. B. (2012). Interactions of nucleolin and ribosomal protein L26 (RPL26) in translational control of human p53 mRNA. J. Biol. Chem. 287, 16467-16476. doi: 10.1074/jbc.M112.349274

Chen, Q., Luo, S., Zhang, Y., and Chen, Z. (2011). Development of a liquid chromatography-mass spectrometry method for the determination of ursolic acid in rat plasma and tissue: application to the pharmacokinetic and tissue distribution study. Anal. Bioanal. Chem. 399, 2877-2884. doi: 10.1007/s00216-011-4651-x

Cheng, Y., Zhao, G., Zhang, S., Nigim, F., Zhou, G., Yu, Z., et al. (2016). AS1411-induced growth inhibition of glioma cells by up-regulation of p53 and down-regulation of Bcl-2 and Akt1 via nucleolin. PLoS ONE 11:e0167094. doi: 10.1371/journal.pone.0167094

Cui, W., Liu, C. X., Zhang, Y. C., Shen, Q., Feng, Z. H., Wang, J., et al. (2019). A novel oleanolic acid derivative HA-19 ameliorates muscle atrophy via promoting protein synthesis and preventing protein degradation. Toxicol. Appl. Pharmacol. 378:114625. doi: 10.1016/j.taap.2019.114625

Dailey, M. M., Miller, M. C., Bates, P. J., Lane, A. N., and Trent, J. O. (2010). Resolution and characterization of the structural polymorphism of a single quadruplex-forming sequence. Nucleic Acids Res. 38, 4877-4888. doi: 10.1093/nar/gkq166

Dumont, N. A., Bentzinger, C. F., Sincennes, M. C., and Rudnicki, M. A. (2015) Satellite cells and skeletal muscle regeneration. Compr. Physiol. 5, 1027-1059. doi: 10.1002/cphy.c140068

Fahling, M., Mrowka, R., Steege, A., Nebrich, G., Perlewitz, A., Persson, P. B., et al. (2006). Translational control of collagen prolyl 4-hydroxylase$\alpha(\mathrm{I})$ gene expression under hypoxia. J. Biol. Chem. 281, 26089-26101. doi: 10.1074/jbc.M604939200

Feng, Z., Shen, Y., Wang, L., Cheng, L., Wang, J., Li, Q., et al. (2011). An oligodeoxynucleotide with promising modulation activity for the proliferation and activation of osteoblast. Int. J. Mol. Sci. 12, 2543-2555. doi: 10.3390/ijms 12042543

Fukada, S. I. (2018). The roles of muscle stem cells in muscle injury, atrophy and hypertrophy. J. Biochem. 163, 353-358. doi: 10.1093/jb/mvy019

Girvan, A. C., Teng, Y., Casson, L. K., Thomas, S. D., Juliger, S., Ball, M. W., et al. (2006). AGRO100 inhibits activation of nuclear 
factor-kappaB (NF-kappaB) by forming a complex with NF-kappaB essential modulator (NEMO) and nucleolin. Mol. Cancer Ther. 5, 1790-1799. doi: 10.1158/1535-7163.MCT-05-0361

Gu, W., Schneider, J. W., Condorelli, G., Kaushal, S., Mahdavi, V., and NadalGinard, B. (1993). Interaction of myogenic factors and the retinoblastoma protein mediates muscle cell commitment and differentiation. Cell 72, 309-324. doi: 10.1016/0092-8674(93)90110-C

Guo, Y., Chen, Y., Wei, Y., Li, H., and Dong, C. (2015). Label-free fluorescent aptasensor for potassium ion using structure-switching aptamers and berberine. Spectrochim. Acta A Mol. Biomol. Spectrosc. 136 Pt C, 1635-1641. doi: 10.1016/j.saa.2014.10.058

Harford, T. J., Kliment, G., Shukla, G. C., and Weyman, C. M. (2017). The muscle regulatory transcription factor $\mathrm{MyoD}$ participates with $\mathrm{p} 53$ to directly increase the expression of the pro-apoptotic Bcl2 family member PUMA. Apoptosis 22, 1532-1542. doi: 10.1007/s10495-017-1423-x

Harford, T. J., Shaltouki, A., and Weyman, C. M. (2010). Increased expression of the pro-apoptotic Bcl2 family member PUMA and apoptosis by the muscle regulatory transcription factor $\mathrm{MyoD}$ in response to a variety of stimuli. Apoptosis 15, 71-82. doi: 10.1007/s10495-009-0428-5

Hartmann, G., Weeratna, R. D., Ballas, Z. K., Payette, P., Blackwell, S., Suparto, I., et al. (2000). Delineation of a CpG phosphorothioate oligodeoxynucleotide for activating primate immune responses in vitro and in vivo. J. Immunol. 164, 1617-1624. doi: 10.4049/jimmunol.164.3.1617

Hou, X., Shen, Y., Zhang, C., Zhang, L., Qin, Y., Yu, Y., et al. (2012). A specific oligodeoxynucleotide promotes the differentiation of osteoblasts via ERK and p38 MAPK pathways. Int. J. Mol. Sci. 13, 7902-7914. doi: 10.3390/ijms13077902

Huang, D. W., Sherman, B. T., and Lempicki, R. A. (2009). Systematic and integrative analysis of large gene lists using DAVID bioinformatics resources. Nat. Protoc. 4, 44-57. doi: 10.1038/nprot.2008.211

Iezzi, S., Di Padova, M., Serra, C., Caretti, G., Simone, C., Maklan, E., et al. (2004). Deacetylase inhibitors increase muscle cell size by promoting myoblast recruitment and fusion through induction of follistatin. Dev. Cell 6, 673-684. doi: $10.1016 / S 1534-5807(04) 00107-8$

Ikebe, J., Umezawa, K., Kamiya, N., Sugihara, T., Yonezawa, Y., Takano, Y., et al. (2011). Theory for trivial trajectory parallelization of multicanonical molecular dynamics and application to a polypeptide in water. J. Comput. Chem. 32, 1286-1297. doi: $10.1002 /$ jcc. 21710

Imenshahidi, M., and Hosseinzadeh, H. (2019). Berberine and barberry (Berberis vulgaris): a clinical review. Phytother. Res. 33, 504-523. doi: 10.1002/ptr.6252

Jia, W., Yao, Z., Zhao, J., Guan, Q., and Gao, L. (2017). New perspectives of physiological and pathological functions of nucleolin (NCL). Life Sci. 186, 1-10. doi: $10.1016 /$ j.lfs.2017.07.025

Juliano, R. L. (2018). Intracellular trafficking and endosomal release of oligonucleotides: What we know and what we don't. Nucleic Acid Ther. 28, 166-177. doi: 10.1089/nat.2018.0727

Kim, D., Pertea, G., Trapnell, C., Pimentel, H., Kelley, R., and Salzberg, S. L. (2013). TopHat2: accurate alignment of transcriptomes in the presence of insertions, deletions and gene fusions. Genome Biol. 14:R36. doi: 10.1186/gb-2013-14-4-r36

Kim, M., Sung, B., Kang, Y. J., Kim, D. H., Lee, Y., Hwang, S. Y., et al. (2015). The combination of ursolic acid and leucine potentiates the differentiation of $\mathrm{C} 2 \mathrm{C} 12$ murine myoblasts through the mTOR signaling pathway. Int. J. Mol. Med. 35, 755-762. doi: 10.3892/ijmm.2014.2046

Klinman, D., Shirota, H., Tross, D., Sato, T., and Klaschik, S. (2008). Synthetic oligodeoxynucleotides as modulators of inflammation. J. Leukoc. Biol. 84, 958-964. doi: 10.1189/jlb.1107775

Krieg, A. M., Yi, A. K., Matson, S., Waldschmidt, T. J., Bishop, G. A., Teasdale, R., et al. (1995). CpG motifs in bacterial DNA trigger direct B-cell activation. Nature 374, 546-549. doi: 10.1038/374546a0

Li, N., Liu, C., Mi, S., Wang, N., Zheng, X., Li, Y., et al. (2012). Simultaneous determination of oleanolic acid, p-coumaric acid, ferulic acid, kaemperol and quercetin in rat plasma by LC-MS-MS and application to a pharmacokinetic study of Oldenlandia diffusa extract in rats. J. Chromatogr. Sci. 50, 885-892. doi: 10.1093/chromsci/bms086

Litchfield, L. M., Riggs, K. A., Hockenberry, A. M., Oliver, L. D., Barnhart, K. G., Cai, J., et al. (2012). Identification and characterization of nucleolin as a COUPTFII coactivator of retinoic acid receptor beta transcription in breast cancer cells. PLoS ONE 7:e38278. doi: 10.1371/journal.pone.0038278
Losfeld, M. E., Khoury, D. E., Mariot, P., Carpentier, M., Krust, B., Briand, J. P., et al. (2009). The cell surface expressed nucleolin is a glycoprotein that triggers calcium entry into mammalian cells. Exp. Cell Res. 315, 357-369. doi: 10.1016/j.yexcr.2008.10.039

Macke, T. J., and Case, D. A. (1998). "Modeling unusual nucleic acid structures," in Molecular Modeling of Nucleic Acids, eds N. B. Leontis and J. SantaLucia (Washington, DC: American Chemical Society), 379-393. doi: 10.1021/bk-1998-0682.ch024

Maier, J. A., Martinez, C., Kasavajhala, K., Wickstrom, L., Hauser, K. E., and Simmerling, C. (2015). ff14SB: Improving the accuracy of protein side chain and backbone parameters from ff99SB. J. Chem. Theory Comput. 11, 3696-3713. doi: 10.1021/acs.jctc.5b00255

Marchildon, F., Lamarche, E., Lala-Tabbert, N., St-Louis, C., and Wiper-Bergeron, N. (2015). Expression of CCAAT/enhancer binding protein beta in muscle satellite cells inhibits myogenesis in cancer cachexia. PLoS ONE 10:e0145583. doi: 10.1371/journal.pone. 0145583

McCormick, R., and Vasilaki, A. (2018). Age-related changes in skeletal muscle: changes to life-style as a therapy. Biogerontology 19, 519-536. doi: 10.1007/s10522-018-9775-3

Nigar, S., Yamamoto, Y., Okajima, T., Shigemori, S., Sato, T., Ogita, T., et al. (2017). Synergistic oligodeoxynucleotide strongly promotes $\mathrm{CpG}$-induced interleukin6 production. BMC Immunol. 18:44. doi: 10.1186/s12865-017-0227-7

Nihashi, Y., Ono, T., Kagami, H., and Takaya, T. (2019a). Toll-like receptor liganddependent inflammatory responses in chick skeletal muscle myoblasts. Dev. Comp. Immunol. 91, 115-122. doi: 10.1016/j.dci.2018.10.013

Nihashi, Y., Umezawa, K., Shinji, S., Hamaguchi, Y., Kobayashi, H., Kono, T., et al. (2019b). Distinct cell proliferation, myogenic differentiation, and gene expression in skeletal muscle myoblasts of layer and broiler chickens. Sci. Rep. 9:16527. doi: 10.1038/s41598-019-52946-4

Norgaard, N. N., Holien, T., Jonsson, S., Hella, H., Espevik, T., Sundan, A., et al. (2010). CpG-oligodeoxynucleotide inhibits Smad-dependent bone morphogenetic protein signaling: effects on myeloma cell apoptosis and in vitro osteoblastogenesis. J. Immunol. 185, 3131-3139. doi: 10.4049/jimmunol.0903605

Novitch, B. G., Mulligan, G. J., Jacks, T., and Lassar, A. B. (1996). Skeletal muscle cells lacking the retinoblastoma protein display defects in muscle gene expression and accumulate in S and G2 phases of the cell cycle. J. Cell Biol. 135, 441-456. doi: 10.1083/jcb.135.2.441

Ou, T. M., Lu, Y. J., Tan, J. H., Huang, Z. S., Wong, K. Y., and Gu, L. Q. (2008). Gquadruplexex: targets in anticancer drug design. ChemMedChem 3, 690-713. doi: $10.1002 / \mathrm{cmdc} .200700300$

Pohar, J., Lainscek, D., Fukui, R., Yamamoto, C., Miyake, K., Jerala, R., et al. (2015). Species-specific minimal sequence motif for oligodeoxyribonucleotides activating mouse TLR9. J. Immunol. 195, 4396-4405. doi: 10.4049/jimmunol.1500600

Porrello, A., Cerone, M. A., Coen, S., Gurtner, A., Fontemaggi, G., Cimino, L., et al. (2000). p53 regulates myogenesis by triggering the differentiation activity of pRb. J. Cell Biol. 151, 1295-1304. doi: 10.1083/jcb.151.6.1295

Quemener, A. M., Bachelot, L., Forestier, A., Donnou-Fournet, E., Gilot, D., and Galibert, M. D. (2020). The powerful world of antisense oligonucleotides: from bench to bedside. Wiley Interdiscip. Rev. RNA 11:e1594. doi: 10.1002/wrna.1594

Ramos, K. S., Moore, S., Runge, I., Tavera-Garcia, M. A., Cascone, I., Courty, J., et al. (2020). The nucleolin antagonist N6L inhibits LINE1 retrotransposon activity in non-small cell lung carcinoma cells. J. Cancer 11, 733-740. doi: $10.7150 /$ jca. 37776

Reyes-Reyes, E. M., Salipur, F. R., Shams, M., Forsthoefel, M. K., and Bates, P. J. (2015). Mechanistic studies of anticancer aptamer AS1411 reveal a novel role for nucleolin in regulating Racl activation. Mol. Oncol. 9, 1392-1405. doi: $10.1016 /$ j.molonc.2015.03.012

Rubin, H. (2003). Cancer cachexia: its correlations and causes. Proc. Natl. Acad. Sci. U.S.A. 100, 5384-5389. doi: 10.1073/pnas.0931260100

Ruijtenberg, S., and van den Heuvel, S. (2016). Coordinating cell proliferation and differentiation: antagonism between cell cycle regulators and cell type-specific gene expression. Cell Cycle 15, 196-212. doi: 10.1080/15384101.2015.1120925

Sackesen, C., van de Veen, W., Akdis, M., Soyer, O., Zumkehr, J., Ruckert, B., et al. (2013). Suppression of B-cell activation and IgE, IgA, IgG1 and IgG4 production by mammalian telomeric oligonucleotides. Allergy 68, 593-603. doi: $10.1111 /$ all.12133 
Shaltouki, A., Freer, M., Mei, Y., and Weyman, C. M. (2007). Increased expression of the pro-apoptotic Bcl2 family member PUMA is required for mitochondrial release of cytochrome $\mathrm{C}$ and the apoptosis associated with skeletal myoblast differentiation. Apoptosis 12, 2143-2154. doi: 10.1007/s10495-007-0135-Z

Shen, Y., Feng, Z., Lin, C., Hou, X., Wang, X., Wang, J., et al. (2012), An oligodeoxynucleotide that induces differentiation of bone marrow mesenchymal stem cells to osteoblasts in vitro and reduces alveolar bone loss in rats with periodontitis. Int. J. Mol. Sci. 13, 2877-2892. doi: 10.3390/ijms13032877

Shinji, S., Nakamura, S., Nihashi, Y., Umezawa, K., and Takaya, T. (2020a). Berberine and palmatine inhibit growth of human rhabdomyosarcoma cells. Biosci. Biotechnol. Biochem. 84, 63-75. doi: 10.1080/09168451.2019.1659714

Shinji, S., Umezawa, K., Nihashi, Y., Nakamura, S., Shimosato, T., and Takaya, T. (2020b). Identification of the myogenetic oligodeoxynucleotides (myoDNs) that promote differentiation of skeletal muscle myoblasts by targeting nucleolin. bioRxiv doi: 10.1101/2020.10.07.330472

Siddiqui-Jain, A., Grand, C. L., Bearss, D. J., and Hurley, L. H. (2002). Direct evidence for a G-quadruplex in a promoter region and its targeting with a small molecule to repress c-MYC transcription. Proc. Natl. Acad. Sci. U.S.A. 99, 11593-11598. doi: 10.1073/pnas.182256799

Soddu, S., Blandino, G., Scardigli, R., Coen, S., Marchetti, A., Rizzo, M. G., et al. (1996). Interference with p53 protein inhibits hematopoietic and muscle differentiation. J. Cell Biol. 134, 193-204. doi: 10.1083/jcb.134.1.193

Szklarczyk, D., Gable, A. L., Lyon, D., Junge, A., Wyder, S., Huerta-Cepas, J., et al. (2019). STRING v11: protein-protein association networks with increased coverage, supporting functional discovery in genome-wide experimental datasets. Nucleic Acids Res. 47, D607-D613. doi: 10.1093/nar/gky1131

Takagi, M., Absalon, M. J., McLure, K. G., and Kastan, M. B. (2005). Regulation of p53 translation and induction after DNA damage by ribosomal protein L26 and nucleolin. Cell 123, 49-63. doi: 10.1016/j.cell.2005.07.034

Takaya, T., Nihashi, Y., Kojima, S., Ono, T., and Kagami, H. (2017). Autonomous xenogenic cell fusion of murine and chick skeletal muscle myoblasts. Anim. Sci. J. 88, 1880-1885. doi: 10.1111/asj.12884

Tang, Z., Qiu, H., Luo, L., Liu, N., Zhong, J., Kang, K., et al. (2017). miR-34b modulates skeletal muscle cell proliferation and differentiation. J. Cell. Biochem. 118, 4285-4295. doi: 10.1002/jcb.26079

Teng, Y., Girvan, A. C., Casson, L. K., Pierce, W. M. Jr, Qian, M., Thomas, S. D., et al. (2007). AS1411 alters the localization of a complex containing protein arginine methyltransferase 5 and nucleolin. Cancer Res. 67, 10491-10500. doi: 10.1158/0008-5472.CAN-06-4206

Tsui, V., and Case, D. A. (2000). Theory and applications of the generalized born solvation model in macromolecular simulations. Biopolymers 56, 275-291. doi: 10.1002/1097-0282(2000)56:4<275::AID-BIP10024>3.0.CO;2-E

Vollmer, J., and Krieg, A. M. (2009). Immunotherapeutic applications of CpG oligodeoxynucleotide TLR9 agonists. Adv. Drug Deliv. Rev. 61, 195-204. doi: 10.1016/j.addr.2008.12.008

Wang, H., Chen, Y., Lu, X. A., Liu, G., Fu, Y., and Luo, Y. (2015). Endostatin prevents dietary-induced obesity by inhibiting adipogenesis and angiogenesis. Diabetes 64, 2442-2456. doi: 10.2337/db14-0528
Wang, J., Wolf, R. M., Caldwell, J. W., Kollman, P. A., and Case, D. A. (2004). Development and testing of a general amber force field. J. Comput. Chem. 25, 1157-1174. doi: $10.1002 /$ jcc. 20035

Wang, T., Chen, C., Larcher, L. M., Barrero, R., and Veedu, R. N. (2019). Three decades of nucleic acid aptamer technologies: lessons learned, progress and opportunities on aptamer development. Biotechnol. Adv. 37, 28-50. doi: 10.1016/j.biotechadv.2018.11.001

Wang, Y., Yamamoto, Y., Shigemori, S., Watanabe, T., Oshiro, K., Wang, X., et al. (2015). Inhibitory/suppressive oligodeoxynucleotide nanocapsules as simple oral delivery devices for preventing atopic dermatitis in mice. Mol. Ther. 23, 297-309. doi: 10.1038/mt.2014.239

Yamamoto, Y., Sugimura, R., Watanabe, T., Shigemori, S., Okajima, T., Nigar, S., et al. (2017). Class A CpG oligodeoxynucleotide priming rescues mice from septic shock via activation of platelet-activating factor acetylhydrolase. Front. Immunol. 8:1049. doi: 10.3389/fimmu.2017.01049

Yang, G., Wan, M., Zhang, Y., Sun, L., Sun, R., Hu, D., et al. (2010). Inhibition of a C-rich oligodeoxynucleotide on activation of immune cells in vitro and enhancement of antibody response in mice. Immunology 131, 501-512. doi: 10.1111/j.1365-2567.2010.03322.x

Yazdian-Robati, R., Bayat, P., Oroojalian, F., Zargari, M., Ramezani, M., Taghdisi, S. M., et al. (2019). Therapeutic applications of AS1411 aptamer, an update review. Int. J. Biol. Macromol. 155, 1420-1431. doi: 10.1016/j.ijbiomac.2019.11.118

Yu, G., and He, Q. Y. (2016). ReactomePA: an R/Bioconductor package for reactome pathway analysis and visualization. Mol. BioSyst. 12, 477-479. doi: 10.1039/C5MB00663E

Zhang, L., Wang, X. H., Wang, H., Du, J., and Mitch, W. E. (2010). Satellite cell dysfunction and impeired IGF-1 signaling cause CKD-induced muscle atrophy. J. Am. Soc. Nephrol. 21, 419-427. doi: 10.1681/ASN.2009060571

Zou, W., Amcheslavsky, A., and Bar-Shavit, Z. (2003). CpG oligodeoxynucleotides modulate the osteoclastogenic activity of osteoblasts via toll-like receptor 9. J. Biol. Chem. 278, 16732-16740. doi: 10.1074/jbc. M212473200

Conflict of Interest: Shinshu University has been assigned the invention of myoDNs by TT, KU, and TS, and Japan Patent Application 2018-568609 has been filed on February 15, 2018.

The remaining authors declare that the research was conducted in the absence of any commercial or financial relationships that could be construed as a potential conflict of interest.

Copyright (๑) 2021 Shinji, Umezawa, Nihashi, Nakamura, Shimosato and Takaya. This is an open-access article distributed under the terms of the Creative Commons Attribution License (CC BY). The use, distribution or reproduction in other forums is permitted, provided the original author(s) and the copyright owner(s) are credited and that the original publication in this journal is cited, in accordance with accepted academic practice. No use, distribution or reproduction is permitted which does not comply with these terms. 